\title{
THE TEICHMÜLLER THEORY OF HARMONIC MAPS
}

\author{
MICHAEL WOLF
}

\section{Introduction}

Let $M$ be a smooth, closed, compact surface of genus $g \geq 2$, let $\mathscr{M}_{-1}$ denote the space of constant curvature -1 metrics on $M$, and let $\sigma|d z|^{2}$ denote a particular element of $\mathscr{M}_{-1}$. The group of diffeomorphisms isotopic to the identity, Diff 0 , acts by pull back on $\mathscr{M}_{-1}$, and we can define the Teichmüller space of genus $g, T_{g}$, to be the quotient space $\mathscr{M}_{-1} / \operatorname{Diff}_{0}$. In studying Teichmüller space, it is natural to pick out a particular hyperbolic metric from each class; here we choose as our representatives the metrics $\rho|d w|^{2}$ which have the property that the map id: $\left(M, \sigma|d z|^{2}\right) \rightarrow\left(M, \rho|d w|^{2}\right)$, which is the identity as a map on $M$, is harmonic as a map of Riemannian manifolds.

Naturally associated to a harmonic map id: $(M, \sigma, z) \rightarrow(M, \rho, w)$ is a quadratic differential $\Phi(\sigma, \rho) d z^{2}$, which is holomorphic with respect to the conformal structure of $\sigma$. This then defines a map $\Phi(\sigma, \cdot): T_{g} \rightarrow \mathrm{QD}(\sigma)$ from the Teichmüller space to the space of holomorphic quadratic differentials on $(M, \sigma)$.

Sampson [17] showed that $\Phi$ is injective and continuous; here we first show (Theorem 3.1) that it is also surjective so that, via $\Phi^{-1}, \mathrm{QD}(\sigma)$ provides global coordinates for $T_{g}$. The rest of this paper is an investigation of those coordinates.

Thurston ([5], [22]) introduced a compactification $\overline{T_{g}^{t h}}$ of $T_{g}$ that differed from the previous compactifications of $T_{g}$ in that the action of the mapping class group (isotopy classes of orientation preserving diffeomorphisms) on $T_{g}$ extended continuously to the boundary $\partial \overline{T_{g}^{t h}}$. From the homeomorphism $\Phi: T_{g} \approx \mathrm{QD}(\sigma)$, we also obtain a compactification $\overline{T_{g}^{h}}(\sigma)$ of $T_{g}$ given by adjoining points at $\infty$ to the rays of the vector space $\mathrm{QD}(\sigma)$. We show (Theorem 4.1) that $\overline{T_{g}^{n}(\sigma)}$ is the Thurston compactification $\overline{T_{g}^{t h}}$, and so $\overline{T_{g}^{h}(\sigma)}$ is independent of the choice of $\sigma$ as a base hyperbolic metric.

Naturally associated (see [10]) to a holomorphic quadratic differential $\Phi d z^{2}$ is a pair of measured foliations on $M$, topological objects. At all points $p \in M$

Received December 1, 1987. The author's research was partially supported by an Alfred P. Sloan Doctoral Dissertation Fellowship. 
where id: $(M, \sigma) \rightarrow(M, \rho)$ is not conformal, there are two distinguished lines in $T_{p} M$ along which the map stretches the most or the least. The foliations of $\Phi(\sigma, \theta) d z^{2}$ integrate the maximal and minimal stretch directions where they are defined, and it is natural to speak of the maximal or minimal stretch foliation. We consider the asymptotics of a family of metrics $\rho_{t}$ whose associated holomorphic quadratic differentials $\Phi\left(\sigma, \rho_{t}\right) d z^{2}$ form a ray $t \Phi_{0} d z^{2}$ $(t \geq 0)$ in $\mathrm{QD}(\sigma)$; for such a family, the foliations of $\Phi\left(\sigma, \rho_{t}\right)$ are constant in $t$, and only the measures of the measured foliations change. The proof of Theorem 4.1 proceeds by showing that, at all but the finitely many points where id: $(M, \sigma) \rightarrow(M, \rho)$ is conformal, the transverse measure associated to the minimal stretch foliation of $\Phi\left(\sigma, \rho_{t}\right) d z^{2}$ is asymptotic to half the amount of the maximal stretch of id: $(M, \sigma) \rightarrow\left(M, \rho_{t}\right)$. So, in a very rough sense, the measured foliations asymptotically approximate their associated metrics, and so a projective class of measured foliations provides a natural limiting point for the family of metrics $\left\{\rho_{t}\right\}$. Moreover, since the measured foliations are topological objects, we expect the boundary of $T_{g}$ which the projective classes represent to be natural, in the sense that it will be independent of the base point $(M, \sigma)$ in $T_{g}$, and that the action of the mapping class group on $T_{g}$ will extend continuously to $\overline{T_{g}^{h}}$.

Working locally, we find that the coordinates $\Phi: T_{g} \rightarrow \mathrm{QD}(\sigma)$ are related to the Weil-Petersson geometry of $T_{g}$. Using these coordinates, we describe a simple recursive method for computing all of the variations of hyperbolic metrics away from $(M, \sigma)$. Using that method, we find that the Hessian of the total energy of a harmonic map, viewed as a function on $T_{g} \times T_{g}$, is the Weil-Petersson metric, and that the fourth variation of the total energy, in the coordinates of $\Phi$, is a multiple of the Weil-Petersson curvature tensor. This method has been used by Jost [12] to derive the Kähler structure and curvature tensor of the Weil-Petersson metric via harmonic maps.

The idea of using harmonic maps to investigate Teichmüller space is not new. Indeed, in 1954, Gerstenhaber and Rauch [8] began a program aimed at proving Teichmüller's Theorem via harmonic maps; their difficulties were finally recently overcome by Reich [14]. Earle and Eells [3] used harmonic maps to portray the space of conformal structures as a trivial fiber bundle over $T_{g}$ with fiber Diff 0 , and to give an explicit section from $T_{g}$ into the space of conformal structures. Finally, while here we show a homeomorphism from $T_{g}$ to $\mathrm{QD}(\sigma)$ by fixing the source surface $(M, \sigma)$ and varying the target surface $(M, \rho)$ over $T_{g}$, Tabak [21], fixing the target surface $(M, \rho)$ and allowing the source surface $(M, \sigma)$ to vary over $T_{g}$, gave a bijection between $T_{g}$ and a class of nonholomorphic quadratic differentials on the target surface $(M, \rho)$. 
The organization of the paper is as follows. In $\S 2$, we give the necessary background, define our terms, and introduce our notation. $\S 3$ is devoted to showing that $\Phi: T_{g} \rightarrow \mathrm{QD}(\sigma)$ is a homeomorphism onto $\mathrm{QD}(\sigma)$. In $\S 4$, we construct the compactification $\overline{T_{g}^{h}(\sigma)}$ and prove Theorem 4.1: $\overline{T_{g}^{h}(\sigma)} \approx \overline{T_{g}^{t h}}$. The discussion of this is broken into subsections: in $\S 4.1$, we describe the motivating example of the asymptotics of harmonic maps between hyperbolic cylinders with boundary; in $\S 4.2$, we state the compactification theorem; and in $\S 4.3$, we prove the compactification theorem using the technical results about asymptotics of harmonic maps which are finally proved in $\S 4.4$. In $\S 5$, we discuss the local geometry of the coordinates $\mathrm{QD}(\sigma)$ : $\S 5.1$ describes our computational method, and $\S 5.2$ discusses the local geometry of the total energy function.

This paper contains the results of the author's thesis. It is a pleasure for him to thank his thesis advisor, Steven Kerckhoff, for all of his help and Halsey Royden for suggesting the problem and for many useful conversations.

Thanks also go to the referee for his careful reading of the manuscript, and his detailed and very useful comments.

\section{Notation and background}

Let $M$ be a fixed, $C^{\infty}$ surface. Consider the metrics $\sigma|d z|^{2}$ and $\rho|d w|^{2}$ on $M$, where $z$ and $w$ are conformal coordinates on $M$. For a Lipschitz map

$$
w:\left(M, \sigma|d z|^{2}\right) \rightarrow\left(M, \rho|d w|^{2}\right)
$$

we define the energy density of $w$ at a point to be

$$
e(w ; \sigma, \rho)=\frac{\rho(w(z))}{\sigma(z)}\left|w_{z}\right|^{2}+\frac{\rho(w(z))}{\sigma(z)}\left|w_{\bar{z}}\right|^{2}
$$

and the total energy

$$
\begin{aligned}
E(w ; \sigma, \rho) & =\int_{M} e(w ; \sigma, \rho) \sigma d z d \bar{z} \\
& =\int_{M} \rho(w(z))\left|w_{z}\right|^{2}+\rho(w(z))\left|w_{\bar{z}}\right|^{2} d z d \bar{z}
\end{aligned}
$$

so that evidently the total energy depends upon the metric of the target surface, but only upon the conformal structure of the source.

A critical point of the energy functional is called a harmonic map; the Euler-Lagrange equation for the energy functional is

$$
\tau(w)=w_{z \bar{z}}+(\log \rho)_{w} w_{z} w_{\bar{z}}=0,
$$

which again depends on the metric of the target but only on the conformal structure of the source. 
Now

$$
\begin{aligned}
w^{*} \rho & =\rho w_{z} \overline{w_{\bar{z}}} d z^{2}+\left(\rho\left|w_{z}\right|^{2}+\rho\left(\left|w_{\bar{z}}\right|^{2}\right) d z d \bar{z}+\overline{\rho w_{z} \overline{w_{\bar{z}}}} d \bar{z}^{2}\right. \\
& =\Phi d z^{2}+\sigma e(w) d z d \bar{z}+\bar{\Phi} d \bar{z}^{2}
\end{aligned}
$$

which defines

$$
\Phi d z^{2}=\left\{\frac{1}{4}\left[\left\|w_{*} \frac{\partial}{\partial x}\right\|_{\rho}^{2}-\left\|w_{*} \frac{\partial}{\partial y}\right\|_{\rho}^{2}\right]-\frac{1}{2} i\left\langle w_{*} \frac{\partial}{\partial x}, w_{*} \frac{\partial}{\partial y}\right\rangle\right\} d z^{2} .
$$

Let

$$
\mathscr{J}(w)=\frac{\rho(w(z))}{\sigma(z)}\left|w_{z}\right|^{2}-\frac{\rho(w(z))}{\sigma(z)}\left|w_{\bar{z}}\right|^{2}
$$

be the Jacobian of $w$. Then if $\mathscr{J}(w)(p) \neq 0$, it is easy to show (see [17]) $w$ harmonic $\Leftrightarrow \tau(w)=0 \Leftrightarrow \Phi d z^{2}$ is a holomorphic quadratic differential. So if $w:(M, \sigma) \rightarrow(M, \rho)$ is holomorphic, we set

$$
\Phi(\sigma, \rho)=\Phi(\rho)=\left(w^{*} \rho\right)^{2,0} .
$$

Define $\mathrm{QD}(\sigma)$ to be the space of holomorphic quadratic differentials on $(M, \sigma)$. Now for any holomorphic quadratic differential $\alpha=\Phi d z^{2} \in \mathrm{QD}(\sigma)$, if $\alpha(p) \neq$ 0 , there is a natural conformal coordinate $\zeta=\xi+i \eta$ so that $\alpha=d \zeta^{2}$ near $p$. The curves $\xi=$ const and $\eta=$ const define a pair of foliations with transverse measures $\mu_{\mathrm{hor}}(\alpha)=\varsigma^{*}|d \eta|$ and $\mu_{\mathrm{vert}}(\alpha)=\varsigma^{*}|d \xi|$, respectively. These easily extend to where $\alpha(p)=0$ to give a pair of transverse (singular) measured foliations associated to $\alpha$, called the horizontal $\left(F_{\text {hor }}(\alpha), \mu_{\text {hor }}(\alpha)\right)$ and vertical $\left(F_{\text {vert }}(\alpha), \mu_{\text {vert }}(\alpha)\right)$ measured foliation of $\alpha$, respectively. In the case where $\Phi d z^{2}$ is determined by a harmonic map, if $z=x+i y$ and $\partial / \partial x$ and $\partial / \partial y$ are tangent to the foliation in a neighborhood (where $\Phi \neq 0$ ), then $\partial / \partial x$ and $\partial / \partial y$ are the directions of maximal and minimal stretch of the differential $d w$, and

$$
\Phi d z^{2}=\frac{1}{4}\left(\left\|w_{*} \frac{\partial}{\partial x}\right\|_{\rho}^{2}-\left\|w_{*} \frac{\partial}{\partial y}\right\|_{\rho}^{2}\right) d z^{2}
$$

with the coefficients of $d z^{2}$ determining the transverse measure to the foliations $x=$ const and $y=$ const.

Almost everything connected with a harmonic map between surfaces can be written in terms of two auxiliary functions; indeed, much of this work can be seen as a fugue on equations of these functions. So we define

$$
\begin{aligned}
& \mathscr{H}=\mathscr{H}(\rho)=\mathscr{H}(\sigma ; \rho)=\frac{\rho(w(z))}{\sigma(z)}\left|w_{z}\right|^{2}, \\
& \mathscr{L}=\mathscr{L}(\rho)=\mathscr{L}(\sigma, \rho)=\frac{\rho(w(z))}{\sigma(z)}\left|w_{\bar{z}}\right|^{2} .
\end{aligned}
$$


Then, defining

$$
\Delta=\frac{4}{\sigma} \frac{\partial^{2}}{\partial z \partial \bar{z}}, \quad K(\sigma)=-\frac{2}{\sigma} \frac{\partial^{2} \log \sigma}{\partial z \partial \bar{z}}, \quad K(\rho)=-\frac{2}{\rho} \frac{\partial^{2} \log \rho}{\partial w \partial \bar{w}}
$$

on $\left(M, \sigma|d z|^{2}\right)$, we find that the Euler-Lagrange equation gives the following equations (see [18]):

(1) $\Delta \log \mathscr{H}=-2 K(\rho) \mathscr{H}+2 K(\rho) \mathscr{L}+2 K(\sigma), \quad$ where $\mathscr{H}(p) \neq 0$

(2) $\quad \Delta \log \mathscr{L}=-2 K(\rho) \mathscr{L}+2 K(\rho) \mathscr{H}+2 K(\sigma), \quad$ where $\mathscr{L}(p) \neq 0$.

Now, we will restrict ourselves to the situation where $K(\sigma)=K(\rho)=-1$. Also, for most of the discussion, $\left(M, \sigma|d z|^{2}\right)$ will be a fixed hyperbolic surface, while $\left(M, \rho|d w|^{2}\right)$ will vary over $T_{g}$. In the situation where the target surface has negative curvature, Eells and Sampson [4] proved the existence of a harmonic map in the homotopy class of the identity, Hartman [9] proved its uniqueness, and, independently, Schoen-Yau [18] and Sampson [17] showed that this map is a diffeomorphism and that $\mathscr{H}>0$.

Finally, we collect some formulas which apply to our source and target surfaces of constant curvature -1 .

(I) The energy density $=e=\mathscr{H}+\mathscr{L}$.

(II) The Jacobian $=\mathscr{J}=\mathscr{H}-\mathscr{L}$.

(III) The norm of the quadratic differential $|\Phi|^{2} / \sigma^{2}=\mathscr{H} \mathscr{L}$.

(IV) The Beltrami differential $\nu=w_{\bar{z}} / w_{z}=\bar{\Phi} / \sigma \mathscr{H}$;

(V) while $|\nu|^{2}=\mathscr{L} / \mathscr{H}$.

(VI) $\Delta \log \mathscr{H}=2 \mathscr{H}-2 \mathscr{L}-2$.

(VII) $\Delta \log \mathscr{L}=2 \mathscr{L}-2 \mathscr{H}-2$ where $\mathscr{L} \neq 0$.

(VIII) $w^{*} \rho=\Phi d z^{2}+\sigma e d z d \bar{z}+\bar{\Phi} d \bar{z}^{2}$.

(IX) $\Phi=\sigma \mathscr{H} \bar{\nu}$.

\section{3. $\Phi: T_{g} \approx \mathrm{QD}(\sigma)$}

Let $M$ be a fixed $C^{\infty}$ surface of genus $g$, and $\sigma$ a fixed hyperbolic metric. We represent each point in $T_{g}$ by a hyperbolic metric; this determines a unique harmonic map homotopic to the identity on $M$ :

$$
w=w(\rho)=w(\sigma, \rho):\left(M, \sigma|d z|^{2}\right) \rightarrow\left(M, \rho|d w|^{2}\right),
$$

and consequently a holomorphic quadratic differential $\Phi \in \mathrm{QD}(\sigma)$ :

$$
\Phi=\Phi(\rho)=\Phi(\sigma, \rho)=\left(w^{*} \rho\right)^{2,0} .
$$

This describes $\Phi$ as a well-defined map $\Phi: T_{g} \rightarrow \mathrm{QD}(\sigma)$. 
Theorem 3.1. $\Phi$ is a homeomorphism from $T_{g}$ onto $\mathrm{QD}(\sigma)$.

Proof. Both $T_{g}$ and $\mathrm{QD}(\sigma)$ are $6 g-6$ dimensional cells, so by Brouwer's invariance of domain, we need only show

(i) $\Phi$ is continuous,

(ii) $\Phi$ is $1-1$,

(iii) $\Phi$ is proper.

Property (i) is clear from the uniqueness of the harmonic map in each homotopy class, and is probably first due to Sampson as reported in EarleEells [3].

Property (ii) is also due to Sampson [17]; we include here for completeness a slightly rearranged proof.

We combine (IV) and (III) to obtain, for $h=\log \mathscr{H}$,

$$
\Delta h=2 e^{h}-2|\Phi|^{2} e^{-h}-2 \text {. }
$$

Consider $\rho_{1}$ and $\rho_{2}$ hyperbolic metrics representing different points in $T_{g}$, and let $\Phi_{i}=\Phi\left(\rho_{i}\right)$.

Suppose $\Phi_{1}=\Phi_{2}=\Phi$. Using the obvious notation, we claim that then $h_{1}=h_{2}$, for if $h_{1}>h_{2}$ somewhere, we can look at a maximum of $h_{1}-h_{2}$ and find that

$$
0 \geq \Delta\left(h_{1}-h_{2}\right)=\left(e^{h_{1}}-e^{h_{2}}\right)-|\Phi|^{2}\left(e^{-h_{1}}-e^{-h_{2}}\right)>0,
$$

since at such a maximum $h_{1}>h_{2}$. So $h_{1} \leq h_{2}$ and, symmetrically, $h_{2} \leq h_{1}$, proving the claim. So $\mathscr{H}_{1}=\mathscr{H}_{2}$, and since $\mathscr{H}_{1} \mathscr{L}_{1}=|\Phi|^{2}=\mathscr{H}_{2} \mathscr{L}_{2}$ and $\mathscr{H}_{1}>0$, we find that $\mathscr{L}_{1}=\mathscr{L}_{2}$. Consequently $e_{1}=e_{2}$, and (VIII) then shows that $w_{1}{ }^{*} \rho_{1}=w_{2}{ }^{*} \rho_{2}$ so that $w_{1} \circ w_{2}^{-1}:\left(M, \rho_{2}\right) \rightarrow\left(M, \rho_{1}\right)$ is an isometry isotopic to the identity.

We are left to prove the properness of $\Phi$. Define $\|\Phi(\rho)\|=\int_{M}|\Phi| d z d \bar{z}$. First prove

Lemma 3.2. $\|\Phi(\rho)\| \rightarrow \infty \Leftrightarrow E(\rho) \rightarrow \infty$.

Proof. Since $\mathscr{H}-\mathscr{L}=\mathscr{J}$ and $\int \mathscr{J} \sigma d z d \bar{z}=-2 \pi \chi$, we have

$$
\begin{aligned}
\int \mathscr{H} \sigma d z d \bar{z}+2 \pi \chi & =\int \mathscr{L} \sigma d z d \bar{z}=\int \Phi \nu d z d \bar{z} \quad \text { since the integrands agree } \\
& \leq \int|\Phi| d z d \bar{z} \quad \text { since } \mathscr{J}>0 \text { implies }|\nu|<1 \\
& =\int \mathscr{H}|\nu| \sigma d z d \bar{z} \quad \text { by (IX) } \\
& \leq \int \mathscr{H} \sigma d z d \bar{z}=\int \mathscr{L} \sigma d z d \bar{z}-2 \pi \chi .
\end{aligned}
$$

So by adding the first two and the last two equalities we find

proving the lemma.

$$
\int e \sigma d z d \bar{z}+2 \pi \chi \leq 2 \int|\Phi| d z d \bar{z} \leq \int e \sigma d z d \bar{z}-2 \pi \chi
$$


So to show the properness of $\Phi(\rho): T_{g} \rightarrow \mathrm{QD}(\sigma)$, we only need to show

Proposition 3.3. $E(\rho): T_{g} \rightarrow \mathbf{R}$ is proper.

Remark. Schoen-Yau [19] proved the properness of $E(\sigma): T_{g} \rightarrow \mathbf{R}$, where $E(\sigma)=E\left(\sigma, \rho_{0}\right)$; here $\rho_{0}$ is a fixed target hyperbolic metric and the source $\sigma$ varies over $T_{g}$.

Proof. We need to show that $B=\left\{\rho \in T_{g}: E(\rho)<K\right\}$ is compact in $T_{g}$. We denote by $l_{\rho}([\gamma])$ the $\rho$-length of the geodesic representative of $[\gamma] \in \pi_{1} M$; it suffices to show that $l_{\rho}([\gamma])<c_{2}(\sigma) K^{1 / 2} l_{\sigma}([\gamma])$. This will follow immediately from the Courant-Lebesgue lemma (see [11, pp. 19-20]): we will show that if $d_{\sigma}\left(x_{1}, x_{2}\right)<\delta<c_{1}(\sigma)$, then

$$
d_{\rho}\left(w\left(x_{1}\right), w\left(x_{2}\right)\right)<4 \sqrt{2} \pi K^{1 / 2}(\log (1 / \delta))^{-1 / 2} .
$$

Before starting that, we rid ourselves of an unnecessary complication; since $w:(M, \sigma) \rightarrow\left(M, \rho_{t}\right)$ is harmonic and homotopic to the identity, the identity map id: $(M, \sigma) \rightarrow\left(M, w^{*} \rho_{t}\right)$ is harmonic. But since $T_{g}$ consists of classes equivalent under the action of $\operatorname{Diff}_{0}$, we might as well have originally chosen $w^{*} \rho_{t}$ to represent the class $\left[\rho_{t}\right] \in T_{g}$. So we will assume for this argument that $\rho_{t}$ was chosen so that id: $(M, \sigma) \rightarrow\left(M, \rho_{t}\right)$ is harmonic.

Now, suppose $d_{\sigma}\left(x_{1}, x_{2}\right)<\delta<\min \left(1, \operatorname{inj}(\sigma)^{2}, 1 /\left(\lambda^{2}\right)\right)$, where $-\lambda^{2}$ is a lower bound for $K(\sigma)$, the curvature of $\sigma$. Of course the restriction on $K(\sigma)$ is not really needed here, but we include it to show the role of curvature. Now introduce coordinates $(r, \theta)$ so that $x_{1}, x_{2} \in B_{\sigma}\left(x_{0}, \delta\right)$ for some $x_{0}$ and $d s_{\sigma}^{2}=d r^{2}+G(r, \theta)^{2} d \theta^{2}$.

Indeed $G(r, \theta)=(1 / \lambda) \sinh \lambda r$ for the metric of constant curvature.

So if $x_{3}, x_{4} \in \partial B_{\sigma}\left(x_{0}, r\right)$, then

$$
d_{\rho}\left(x_{3}, x_{4}\right) \leq \int_{0}^{2 \pi}\left\|\frac{\partial}{\partial \theta}\right\|_{\rho} d \theta \leq 2 \pi\left(\int_{0}^{2 \pi}\left\|\frac{\partial}{\partial \theta}\right\|_{\rho}^{2} d \theta\right)^{1 / 2} .
$$

Let $A=A\left(x_{0}, \delta, \delta^{1 / 2}\right)$ be the annulus centered at $x_{0}$ of inner radius $\delta$ and outer radius $\delta^{1 / 2}$, distances being measured in the $\sigma$ metric.

$$
\begin{aligned}
K & \geq E(\sigma, \rho) \geq \iint e \sigma d z d \bar{z} \\
& =\frac{1}{2} \iint_{A}\left\|\frac{\partial}{\partial r}\right\|_{\rho}^{2}+\frac{1}{G^{2}}\left\|\frac{\partial}{\partial \theta}\right\|_{\rho}^{2} G d r d \theta \\
& \geq \frac{1}{2} \iint_{A}\left\|\frac{\partial}{\partial \theta}\right\|_{\rho}^{2} d \theta \frac{d r}{G(r)}
\end{aligned}
$$


Since $G(r, \theta)=(1 / \lambda) \sinh \lambda r$, for $r<\delta^{1 / 2}<1 / \lambda$ we find that $G(r)<2 r$ and therefore

$$
\int_{\delta}^{\delta^{1 / 2}} \frac{d r}{G(r)}>\frac{1}{4} \log \frac{1}{\delta}
$$

So we can find some $r \in\left(\delta, \delta^{1 / 2}\right)$ with

$$
\int_{0}^{2 \pi}\left\|\frac{\partial}{\partial \theta}\right\|_{\rho}^{2} d \theta \leq 8 K\left(\log \frac{1}{\delta}\right)^{-1} .
$$

For that choice of $r$ in (3.1), inequalities (3.2) and (3.3) yield

$$
d_{\rho}\left(x_{1}, x_{2}\right) \leq d_{\rho}\left(x_{3}, x_{4}\right) \leq 4 \sqrt{2} \pi K^{1 / 2}\left(\log \frac{1}{\delta}\right)^{-1 / 2} .
$$

Remark. Classically, much of Teichmüller theory has been based on the study of the Beltrami equation $w_{\bar{z}}=\mu w_{z}$, where the parameter $\mu$ belonged to some (typically) infinite dimensional function or tensor space; an appropriate equivalence relation is often required to pass from the parameter or solution space to Teichmüller space. Theorem 1.1 implies that we can solve

$$
\Delta \log \mathscr{H}=2 \mathscr{H}-2|\Phi|^{2} /\left(\sigma^{2} \mathscr{H}\right)-2
$$

on a Riemann surface $(M, \sigma)$ for any parameter $\Phi \in \mathrm{QD}(\sigma)$; then $d s_{\rho(\Phi)}^{2}=$ $\Phi d z^{2}+\sigma\left(\mathscr{H}+|\Phi|^{2} /\left(\sigma^{2} \mathscr{H}\right)\right) d z d \bar{z}+\Phi d \bar{z}^{2}$ is a hyperbolic representative of a point in $T_{g}$, determined by and determining $\Phi$ uniquely. So we can interpret Theorem 1.1 as saying that Teichmüller space can be studied through (3.4), where the parameter space is finite dimensional and no equivalence relation needs to be applied.

We will study the variational theory of this equation in $\S 5$.

\section{The harmonic maps compactification of $T_{g}$}

Since $\Phi: T_{g} \rightarrow \mathrm{QD}(\sigma)$ is a homeomorphism onto $\mathrm{QD}(\sigma)$, the vector space $\mathrm{QD}(\sigma)$ provides coordinates for $T_{g}$ centered at $\sigma$, as well as some distinguished submanifolds in $T_{g}$. In particular, let $\Phi_{0} \in \mathrm{QD}(\sigma)$ be a nonzero holomorphic quadratic differential on $(M, \sigma)$. Then $t \Phi_{0}, t \in \mathbf{R}, t \geq 0$, is a ray in $\mathrm{QD}(\sigma)$ with vertex at 0 . Via the homeomorphism $\Phi, t \Phi_{0}$ determines a family of metrics $\rho_{t}$ with $\rho_{0}=\sigma$ so that $\rho_{t}$ leaves every compact set of $\partial T_{g}$; on the other hand, $t \Phi_{0}$ also determines a family of measured foliations on $M$ as described in $\S 2$. In this section we describe an asymptotic relationship between the family of hyperbolic metrics $\left\{\rho_{t}\right\}$ and the family of vertical measured foliations $\left(F_{\text {vert }}\left(t \Phi_{0}\right), \mu_{\text {vert }}\left(t \Phi_{0}\right)\right)$ 
4.1. An example. We begin with the example of $M$ being a hyperbolic cylinder with boundary in which both families can be explicitly described. Specifically, we realize $(M, \sigma)$ as the rectangle $[-1,1] \times[0,1]$ in the $z$-plane with metric $d s^{2}=d x^{2}+d y^{2}$ (identifying $[-1,1] \times\{0\}$ with $[-1,1] \times\{1\}$ to obtain a cylinder $)$, and $\left(M, \rho_{t}\right)$ as the rectangle $\left[-\cosh ^{-1} t, \cosh ^{-1} t\right] \times[0,1]$ in the $w$-plane with the metric $d s^{2}=d u^{2}+\left[\left(\cosh ^{2} u\right) / t^{2}\right] d v^{2}$ (identifying $\left[\cosh ^{-1} t,-\cosh ^{-1} t\right] \times\{0\}$ and $\left[\cosh ^{-1} t,-\cosh ^{-1} t\right] \times\{1\}$ to obtain a cylinder). Then $\rho_{t}$ has constant curvature -1 , while $\sigma$ has vanishing curvature, which is of no consequence for us since, as we noted earlier, only the conformal structure of the source manifold is important for the harmonic map equation. The metric $\rho_{t}$ is normalized so that the curves $u= \pm \cosh ^{-1} t$ have $\rho_{t}$-length 1 , while the curve $u=0$ has $\rho_{t}$-length $\varepsilon=1 / t$.

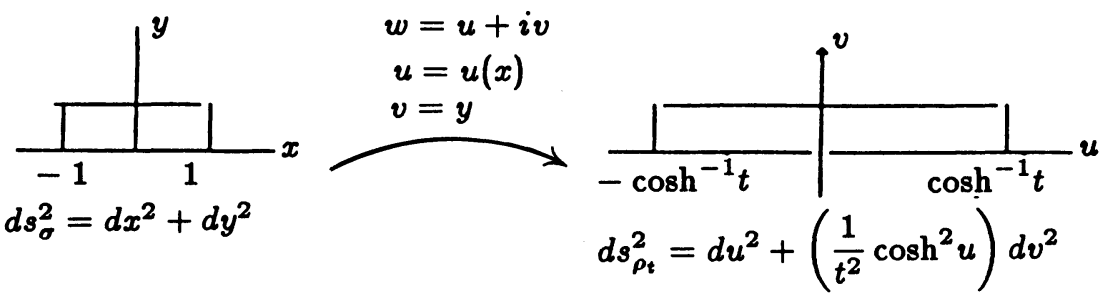

FIGURE 1

We consider maps $w:(M, \sigma) \rightarrow\left(M, \rho_{t}\right)$ with the boundary condition that $w( \pm 1, y)=\left( \pm \cosh ^{-1} t, y\right)$; because of the symmetry of the situation, we can describe the harmonic map in this class as $w=u+i v, v=y, u=u(x)$. We easily compute the Euler-Lagrange equation in this one-dimensional case to be

$$
u^{\prime \prime}(x)=\frac{1}{2 t^{2}} \sinh 2 u, \quad u(0)=0, \quad u(1)=\cosh ^{-1} t .
$$

We consider the solution

$$
u=x \cosh ^{-1} t+\beta(x), \quad \beta(0)=0=\beta(1),
$$

so that $\beta$ represents the deviation of the map from being affine.

It is not hard to show that $\beta \rightarrow 0$ as $t \rightarrow \infty$. So for $t$ very large, $w=u+i v$ deviates very little from an affine stretch. We can rephrase this in terms of vertical measured foliations as follows. The holomorphic quadratic differential $\Phi_{t}$ associated to $\rho_{t}$ is given by

$$
\Phi_{t}=\frac{1}{4}\left(\left\|u_{*} \frac{\partial}{\partial x}\right\|_{\rho_{t}}^{2}-\left\|v_{*} \frac{\partial}{\partial y}\right\|_{\rho_{t}}^{2}\right) d z^{2} .
$$


It has a vertical foliation given by the curves $x=$ const in the $z$-plane, and a transverse measure of

$$
\frac{1}{2}\left(\left\|u_{*} \frac{\partial}{\partial x}\right\|_{\rho_{t}}^{2}-\left\|v_{*} \frac{\partial}{\partial y}\right\|_{\rho_{t}}^{2}\right)^{1 / 2}|d x|,
$$

the coefficient of which, being both real and holomorphic, is a constant. So given a horizontal line segment $\gamma: a \leq x \leq b$, the vertical measure of this segment is

$$
\begin{aligned}
i\left(F_{\text {vert }}\left(\Phi_{t}\right), \gamma\right) & =\int_{a}^{b} \frac{1}{2}\left(\left\|u_{*} \frac{\partial}{\partial x}\right\|_{\rho_{t}}^{2}-\left\|v_{*} \frac{\partial}{\partial y}\right\|_{\rho_{t}}^{2}\right)^{1 / 2} d x \\
& =\int_{a}^{b} \frac{1}{2}\left\|u_{*} \frac{\partial}{\partial x}\right\|_{\rho_{t}}\left(1-\frac{\left\|v_{*} \partial / \partial y\right\|_{\rho_{t}}^{2}}{\left\|u_{*} \partial / \partial x\right\|_{\rho_{t}}^{2}}\right) d x
\end{aligned}
$$

On the other hand, the $\rho_{t}$-length of $w(\gamma)$ is

$$
l_{\rho_{t}}(w(\gamma))=\int_{a}^{b}\left\|u_{*} \frac{\partial}{\partial x}\right\|_{\rho_{t}} d x,
$$

so that, since $\left\|v_{*} \partial / \partial y\right\|_{\rho_{t}} /\left\|u_{*} \partial / \partial x\right\|_{\rho_{t}} \rightarrow 0$, we find

$$
\frac{i\left(F_{\text {vert }}\left(\Phi_{t}\right), \gamma\right)}{\frac{1}{2} l_{\rho_{t}}(w(\gamma))} \rightarrow 1
$$

So the import of $w$ deviating very little from an affine map is now clear: topologically (the minimal stretch), vertical lines that are equally spaced with respect to the $\rho_{t}$ metric, will, when pulled back to the $z$-plane, converge to vertical lines that are equally spaced with respect to a measure for the vertical foliation. Moreover, we could go on to show that given any arc $\gamma$, the ratio of half its $\rho_{t}$-length to its measure against the vertical foliation of $\Phi_{t}$ goes to unity; this means that the asymptotic ratio of $\rho_{t}$ lengths of two $\operatorname{arcs} \gamma_{1}$ and $\gamma_{2}$ is determined by the ratio of their measures against any $\Phi_{t}$ foliation, which is a topological object on the cylinder.

4.2. Statement of the compactification theorem. We want to generalize this situation to compact surfaces of genus $g \geq 2$; in order to make a precise statement we first describe the Thurston compactification $\overline{T_{g}^{t h}}$ of Teichmüller space. (A good reference for this is [5].) Let $\mathscr{S}$ be the set of isotopy classes of simple, homotopically nontrivial curves on $M$, let $\mathscr{M F}$ be the space of measured foliations up to isotopy and Whitehead moves, and let $\mathscr{M F ^ { * }}$ be the space of nontrivial measured foliations on $M$ up to isotopy and Whitehead moves. Let $\pi: \mathbf{R}_{+}^{\mathscr{S}}-0 \rightarrow P\left(\mathbf{R}_{+}^{\mathscr{S}}\right)$ be the natural projection onto the projective space of functionals $P\left(\mathbf{R}_{+}^{\mathscr{S}}\right)$. 
Let $\rho$ be a hyperbolic metric on $M$; then for each class $[\gamma] \in \mathscr{S}$, there is a unique $\rho$-geodesic $\gamma \in[\gamma]$ representing $[\gamma]$ on $(M, \rho)$, and we can measure its $\rho$-length, $l_{\rho}(\gamma)$. Thus we have maps

$$
\begin{gathered}
l: T_{g} \rightarrow \mathbf{R}_{+}^{\mathscr{S}}-0, \\
\rho \mapsto l_{\rho}: \mathscr{S}^{\rightarrow} \mathbf{R}_{+}
\end{gathered}
$$

and, in fact,

$$
\pi \circ l: T_{g} \rightarrow P\left(\mathbf{R}_{+}^{\mathscr{S}}\right)
$$

is an injection (see [5]). We can also assign a functional to each nonzero measured foliation $(\mathscr{F}, \mu) \in \mathscr{M F}^{*}$ : we define the intersection number of $[\gamma]$ with $(\mathscr{F}, \mu)$ to be

$$
i(\mathscr{F}, \mu ;[\gamma])=\inf _{\gamma \in[\gamma]} \int_{\gamma} \mu,
$$

the infimum of $\mu$-transverse measures of representations of $[\gamma]$. So we get another map

$$
\begin{gathered}
I: \mathscr{M F} \mathscr{F}^{*} \rightarrow \mathbf{R}_{+}^{\mathscr{S}}-0, \\
(\mathscr{F}, \mu) \mapsto(\mathscr{F}, \mu): \mathscr{S} \rightarrow \mathbf{R}_{+}
\end{gathered}
$$

which is also an injection; we identify $\mathscr{M F}^{*}$ with its image in $\mathbf{R}_{+}^{\mathscr{S}}$. Finally we define the space of projective measured foliations to be $\mathscr{P F}=\pi \circ I\left(\mathscr{M} \mathscr{F}^{*}\right) \subset$ $P\left(\mathbf{R}_{+}^{\mathscr{S}}\right)$.

The Thurston compactification is $\overline{T_{g}^{t h}}=\pi \circ l\left(T_{g}\right) \cup \mathscr{P} \mathscr{F}$ with a topology induced from the topology of $P \mathbf{R}_{+}^{\mathscr{S}}$ (see [5]). Thurston proves that $\mathscr{P} \mathscr{F} \approx S^{6 g-7}$ and that $\overline{T_{g}^{t h}} \subset P\left(\mathbf{R}_{+}^{\mathscr{S}}\right)$ is a compact manifold with boundary, homeomorphic to a closed ball with boundary sphere $\mathscr{P} \mathscr{F}$.

By construction, Thurston's compactification of $T_{g}$ does not depend on a choice of base point within $T_{g}$, and since the mapping class group $\Gamma_{g}$ acts continuously on $\mathscr{M F}$, its action on $T_{g}$ extends to $\overline{T_{g}^{t h}}$. This compactification should be contrasted with Teichmüller's compactification of $T_{g}$ : by attaching points at $\infty$ to Teichmüller's embedding of $T_{g}$ into $\mathrm{QD}(\sigma)$ we obtain a compactification $\overline{T_{g}^{T}}(\sigma)$ which does depend on a choice of base point in $T_{g}$, and on which the action of the mapping class group does not extend continuously to $\partial \overline{T_{g}^{T}}(\sigma)[13]$.

Define, recalling that $\|\Phi\|=\int_{M}|\Phi| d z d \bar{z}$,

$$
\begin{aligned}
& \mathrm{BQD}(\sigma)=\{\Phi \in \mathrm{QD}(\sigma):\|\Phi\|<1\} \\
& \mathrm{SQD}(\sigma)=\{\Phi \in \mathrm{QD}(\sigma):\|\Phi\|=1\} \\
& \overline{\mathrm{BQD}(\sigma)}=\mathrm{BQD} \cup \mathrm{SQD} \quad \text { with the } \mathrm{QD}(\sigma) \text { topology. }
\end{aligned}
$$


Now we define a map

$$
\tilde{\Phi}: T_{g} \rightarrow \mathrm{BQD}(\sigma)
$$

given by $\tilde{\Phi}(\rho)=4 \Phi(\rho) /(1+4\|\Phi(\rho)\|)$. Clearly $\tilde{\Phi}$ is a homeomorphism onto $\operatorname{BQD}(\sigma)$. Using $\tilde{\Phi}$ to identify $T_{g}$ with $\operatorname{BQD}(\sigma)$, we define a new compactification of $T_{g}$,

$$
\overline{T_{g}^{h}(\sigma)}=T_{g} \cup \operatorname{SQD}(\sigma)=\overline{\mathrm{BQD}}(\sigma) .
$$

The goal of the rest of this section is to prove

Theorem 4.1. $\overline{T_{g}^{h}(\sigma)} \approx \overline{T_{g}^{t h}}$.

Corollary 4.2. $\frac{T_{g}^{h}}{(\sigma)}=\bar{T}_{g}^{h}$ is independent of the choice of base point $\sigma$. The action of the mapping class group $\Gamma_{g}$ on $T_{g}$ extends continuously to an action on $\overline{T_{g}^{h}} \cdot \overline{T_{g}^{h}} \not \overline{T_{g}^{T}}$.

Remark. Theorem 4.1 provides an internal ray structure to Thurston's compactification of $T_{g}$ from each point $\sigma$ of $T_{g}$.

The proof of Theorem 4.1 depends on a description of the asymptotics of the Beltrami differentials for the harmonic maps.

Proposition 4.3.. Let $\Phi_{0} \in \operatorname{SQD}(\sigma)$. Let $\left\{\rho_{t}\right\}=\Phi^{-1}\left\{t \Phi_{0}, t>0\right\}$ be the family of hyperbolic metrics determined by $t \Phi_{0}$. Let $\nu(t) \in \operatorname{Belt}(\sigma)$ be the family of Beltrami differentials determined by the harmonic maps $w(t):(M, \sigma) \rightarrow$ $\left(M, \rho_{t}\right)$, i.e.,

$$
\nu(t)=w(t)_{\bar{z}} / w(t)_{z}
$$

Then if $\Phi_{0}(p) \neq 0,|\nu(t)(p)|^{2} \uparrow 1$.

We postpone the proof of this proposition until $\S 4.4$.

By construction, the foliations of $\Phi\left(\rho_{t}\right)=t \Phi_{0}$ are fixed for all $t$; only the measures of the measured foliations associated to $t \Phi_{0}$ change, and Proposition 4.3 can be understood as describing the asymptotics of these measures as $t \rightarrow \infty$. Towards this end, let $\partial / \partial x, \partial / \partial y$ be an orthonormal frame field on $(M, \sigma)$ (away from the zeros of $\Phi_{0}$ ) tangent to the horizontal and vertical foliations, respectively. Then, by construction, $\partial / \partial x$ and $\partial / \partial y$ are also the directions of maximum and minimum stretch of the differential map $d w(t)$. So in these coordinates,

$$
\begin{gathered}
t \Phi_{0}=\frac{1}{4}\left[\left\|\frac{\partial}{\partial x}\right\|_{\rho_{t}}^{2}-\left\|\frac{\partial}{\partial y}\right\|_{\rho_{t}}^{2}\right] d z^{2} \\
|\mu(t)|=\frac{1-\|\partial / \partial y\|_{\rho_{t}} /\|\partial / \partial x\|_{\rho_{t}}}{1+\|\partial / \partial y\|_{\rho_{t}} /\|\partial / \partial x\|_{\rho_{t}}}
\end{gathered}
$$

Let $\|v\|_{\Phi\left(\rho_{t}\right)}$ be the norm given by the (singular) flat metric $d s_{\Phi\left(\rho_{t}\right)}^{2}=$ $t\left|\Phi_{0}\right||d z|^{2}$ to a vector $v \in T_{p} M, \Phi_{0}(p) \neq 0$. Then

$$
\left\|\frac{\partial}{\partial x}\right\|_{\Phi\left(\rho_{t}\right)}^{2}=\frac{1}{4}\left(\left\|\frac{\partial}{\partial x}\right\|_{\rho_{t}}^{2}-\left\|\frac{\partial}{\partial y}\right\|_{\rho_{t}}^{2}\right) .
$$


Thus we can rephrase Proposition 4.3 as

Proposition 4.4. Let $\rho_{t}$ be defined as in Proposition 2.3. Then

(i) $\left(\|\partial / \partial y\|_{\rho_{t}} /\|\partial / \partial x\|_{\rho_{t}}\right) \downarrow 0$ for all points $p$ with $\Phi_{0}(p) \neq 0$.

(ii) $1>\left(2\|\partial / \partial x\|_{\Phi\left(\rho_{t}\right)} /\|\partial / \partial x\|_{\rho_{t}}\right) \uparrow 1$ for all points $p$ with $\Phi_{0}(p) \neq 0$.

Corollary 4.5. Let $l_{\rho_{t}}(\gamma)$ denote the $\rho_{t}$-length of the arc $\gamma$.

(a) Then $\left\|\partial /\left.\partial y\right|_{p}\right\|_{\rho_{t}} \rightarrow 0$ at almost all points $p$.

(b) If $\gamma$ is a closed arc of the horizontal foliation of $t \Phi_{0}$, and $\gamma$ does not contain a zero of $\Phi_{0}$, then there are constants $C_{0}$ and $C_{1}$ depending only on $\gamma$ so that

$$
0<C_{0}<l_{\rho_{t}}(\gamma) t^{-1 / 2}<C_{1}<\infty
$$

(c) If $\gamma$ is an arc of the vertical foliation of $t \Phi_{0}$, then

$$
l_{\rho_{t}}(\gamma) t^{-1 / 2} \rightarrow 0 \quad \text { as } t \rightarrow \infty
$$

We postpone the proof of Proposition 4.3 and Corollary 4.5 until $§ 4.4$.

4.3. Proof of the compactification theorem. Define

$$
\begin{aligned}
\beta: \operatorname{BQD}(\sigma) & \rightarrow M \mathscr{F} \subset \mathbf{R}_{+}^{\mathscr{S}} \\
\tilde{\Phi} & \mapsto \beta(\tilde{\Phi})
\end{aligned}
$$

so that $\beta(\tilde{\Phi})$ is the vertical measured foliation (as an element of $\mathbf{R}_{+}^{\mathscr{S}}$ ) of

$$
4 \Phi=\frac{4 \tilde{\Phi}}{1-\|\tilde{\Phi}\|} .
$$

By a theorem of Hubbard and Masur [10], $\beta$ is a homeomorphism of $\operatorname{BQD}(\sigma)$ onto $\mathscr{M F} \subset \mathbf{R}_{+}^{\mathscr{S}}$. As a consequence, we find that if $\left\|\tilde{\Phi}_{n}\right\| \rightarrow 1$, then $\tilde{\Phi}_{n}$ converges in $\operatorname{BQD}(\sigma) \Leftrightarrow \pi \circ \beta \tilde{\Phi}_{n}$ converges in $P\left(\mathbf{R}_{+}^{\mathscr{S}}\right)$.

We will understand the notation $\beta(\Phi)$ to mean

$$
\dot{\beta(\tilde{\Phi}}(\Phi))=\beta(4 \Phi /(1+4\|\Phi\|)) .
$$

Because $\beta \Phi$ and $\beta(\Phi /\|\Phi\|)$ represent measured foliations which have the same underlying foliation and differ only their transverse measures, for $[\gamma] \in$ $\mathscr{S}$, we have

$$
i(\beta \Phi,[\gamma])=\|\Phi\|^{1 / 2} i(\beta(\Phi /\|\Phi\|),[\gamma])
$$

Lemma 4.6. For all classes $[\gamma] \in \mathscr{S}$, there exist $k_{0}=k_{0}(\|\Phi(\rho)\|,[\gamma])$ and $\eta=\eta(\|\Phi(\rho)\|,[\gamma])$, both depending on $\|\Phi(\rho)\|$ and $[\gamma]$, so that

$$
k_{0} i(\beta \Phi(\rho),[\gamma])+\eta \geq l_{\rho}([\gamma]) \geq i(\beta \Phi(\rho),[\gamma]),
$$

where $k_{0} \downarrow 1$ and $\eta\|\Phi(\rho)\|^{-1 / 2} \rightarrow 0$ as $\|\Phi(\rho)\| \rightarrow \infty$. 
Proof. First we prove $l_{\rho}([\gamma]) \geq i(\beta \Phi(\rho),[\gamma])$. Let $\gamma:[0,1] \rightarrow M$ be the constant speed parametrization of a $\rho_{t}$-geodesic. Let $\dot{\gamma}_{h}, \dot{\gamma}_{v}$ denote the horizontal and vertical components of $\dot{\gamma}$ relative to the horizontal and vertical foliations. Then

$$
\begin{aligned}
l_{\rho}([\gamma]) & =l_{\rho}=\int_{0}^{1}\left(\left\|\dot{\gamma}_{h}\right\|_{\rho}^{2}+\left\|\dot{\gamma}_{v}\right\|_{\rho}^{2}\right)^{1 / 2} d s \\
& \geq \int\left\|\dot{\gamma}_{h}\right\|_{\rho} d s \\
& \geq \int\left\|\dot{\gamma}_{h}\right\|_{4 \Phi(\rho)} d s \quad \text { by Proposition 4.4(ii) } \\
& =i(\beta \Phi(\rho), \gamma) \geq i(\beta \Phi(\rho),[\gamma]) .
\end{aligned}
$$

Next we prove $k_{0} i(\beta \Phi(\rho),[\gamma])+\eta \geq l_{\rho}([\gamma])$. This estimate requires a little preparation.

First, we claim that for every $[\gamma] \in \mathscr{S}$ there are numbers $\varepsilon([\gamma]), K([\gamma])$, and $L([\gamma])$ so that for every $\Phi \in \operatorname{SQD}(\sigma)$, there is a representative $\gamma_{\Phi} \in[\gamma]$ so that

(i) all of the unbroken segments of $\gamma$ lie along leaves of either the horizontal or vertical foliations,

(ii) all horizontal segments of $\gamma_{\Phi}$ avoid a neighborhood of $\sigma$-radius $\varepsilon([\gamma])$ of the zeros of $\Phi$,

(iii) $i\left(\beta \Phi, \gamma_{\Phi}\right)=i(\beta \Phi,[\gamma])$,

(iv) $i\left(\beta(-\Phi), \gamma_{\Phi}\right)<K([\gamma])$, i.e., the total length of the vertical arcs, when measured in the metric $|\Phi||d z|^{2}$, is uniformly bounded by $K([\gamma])$; also $\gamma_{\Phi}$ contains fewer than $L([\gamma])$ vertical arcs through zeros of $\Phi$.

We first prove this statement for a neighborhood of an element $\Phi_{0} \in$ $\operatorname{SQD}(\sigma)$. Now the zeros of $\Phi_{0}$, say $\left\{p_{1}, \cdots, p_{n}\right\}$, are isolated; then there is a $\delta\left(\Phi_{0}\right)$ so that each disk $B_{2 \delta\left(\Phi_{0}\right)}\left(p_{i}\right)$ of $\Phi_{0}$-radius $2 \delta\left(\Phi_{0}\right)$ around a zero contains only one zero of $\Phi_{0}$, is topologically a disk, and is separated from any other such disk, $B_{2 \delta\left(\Phi_{0}\right)}\left(p_{j}\right)$, by a $\Phi_{0}$-distance of at least $2 \delta\left(\Phi_{0}\right)$. We also assume that

$$
\left.\delta\left(\Phi_{0}\right)<\left(\min _{\Phi \in \mathrm{SQD}(\sigma)} \text { (injectivity radius of }|\Phi||d z|^{2}\right)\right) /(8 g-7) .
$$

We then can consider a small neighborhood $N$ of $\Phi_{0}$ in $\operatorname{SQD}(\sigma)$ with the properties that if $\Phi \in N$, then (a) the natural correspondence between the connected components of $\bigcup_{\Phi_{0}(p)=0} B_{\delta\left(\Phi_{0}\right)}(p)$ and the connected components of $\bigcup_{\Phi(p)=0} B_{\delta\left(\Phi_{0}\right)}(p)$ is a bijection, (b) each connected component $C$ of $\bigcup_{\Phi(p)=0} B_{\delta\left(\Phi_{0}\right)}(p)$ is a topological disk separated from the other connected components by a $\Phi$-distance of at least $\delta\left(\Phi_{0}\right)$, and (c) a connected vertical arc in $C$ has $\Phi$-length of at most $K\left(\Phi_{0}\right)$. (The point here is that in $N$, a 
deformation neighborhood of $\Phi_{0}$, we permit high order zeros of $\Phi_{0}$ to split into lower order zeros so near each other that a neighborhood of them is still topologically trivial, but we do not allow the neighborhoods of the isolated zeros of $\Phi_{0}$ to migrate too near each other.)

Now we construct $\gamma_{\Phi}$. We start with a geodesic $\Gamma_{\Phi}$ (of the metric $|\Phi||d z|^{2}$; see [20, Chapter V, especially Theorem 18.4]) which, by virtue of being quasitransversal, satisfies condition (iii). Outside of $\bigcup_{\Phi(p)=0} B_{\delta\left(\Phi_{0}\right)}(p), \Gamma_{\Phi}$ is locally a line of constant argument in the plane, and we can replace it there by a step-curve of arcs of the vertical and horizontal foliation, whose horizontal measure agrees with the original horizontal measure, and whose $\Phi$-length of vertical arc is less than the original $\Phi$-length of the curve. Inside one of the connected components of $\bigcup_{\Phi(p)=0} B_{\delta\left(\Phi_{0}\right)}(p)$, say $C$, we notice that there are at most $4 g-4$ zeros of $\Phi$ and $12 g-12$ connected components of vertical arcs which have a zero of $\Phi$ as an endpoint (the latter we call critical vertical arcs). Let $\tilde{\gamma}_{\Phi}$ be a connected component of $\Gamma_{\Phi} \cap C$. (There are only finitely many such components: since $\operatorname{SQD}(\sigma)$ is compact, the minimal $\Phi$-length of $[\gamma]$ is bounded above, while the injectivity radius is bounded below and away from $(8 g-7) \delta\left(\Phi_{0}\right)$. So, because of the convexity of $B_{\delta\left(\Phi_{0}\right)}(p)$ in the $\Phi_{0}$ metric, an arc of $\Gamma_{\Phi}$ can pass through $C$ only as many times as there are zeros of $\Phi$ in $C$, and after doing so, it must travel at least $\delta\left(\Phi_{0}\right)$ before returning to $C$.) Then $\tilde{\gamma}_{\Phi}$ will either cross or contain each critical vertical arc at most once, since both $\tilde{\gamma}_{\Phi}$ and the critical arcs are $\Phi$-geodesics. We can then replace the subarcs between such intersections with a $\Phi$-polygonal curve, all of whose horizontal subarcs are outside of $C$, by a process of dragging the horizontal measure out of $C$ on vertical arcs as illustrated in Figure 2. This process will preserve the horizontal measure of $\tilde{\gamma}_{\Phi}$ while adding only a finite amount of vertical measure and a bounded number of critical vertical arcs, the last two because we have assumed that all connected vertical arcs have $\Phi$-length bounded by $K\left(\Phi_{0}\right)$, and we use fewer than $2(4 g-4)^{2}$ of these arcs in the process of "dragging" the horizontal measure out of $C$.

Since now all of the horizontal arcs avoid a neighborhood of $\Phi$-radius $\delta\left(\Phi_{0}\right)$ of the zeros of $\Phi$, the horizontal arcs avoid a neighborhood of $\sigma$-radius $\varepsilon\left(\Phi_{0}\right)$ of the zeros of $\Phi$. So we have accomplished (i)-(iv) in a neighborhood $N$ of $\Phi_{0}$. Since $\operatorname{SQD}(\sigma)$ is compact we need only a finite number of such neighborhoods to cover $\operatorname{SQD}(\sigma)$, and we find we can assume (i)-(iv) for all $\Phi \in \operatorname{SQD}(\sigma)$.

Let $M_{\varepsilon([\gamma])}(\rho)=M \sim \bigcup_{\Phi(\rho)(p)=0} B_{\varepsilon([\gamma])}(p)$ where $B_{\varepsilon([\gamma])}(p)$ is now the open ball of $\sigma$-radius $\varepsilon([\gamma])$ around $p$. Let $\partial / \partial x$ denote a vector field on a coordinate patch on $M$, tangent to the horizontal (maximal stretch) foliation, undefined in very small neighborhoods, say of $\sigma$-radius $\varepsilon([\gamma]) / 10$, of the zero 

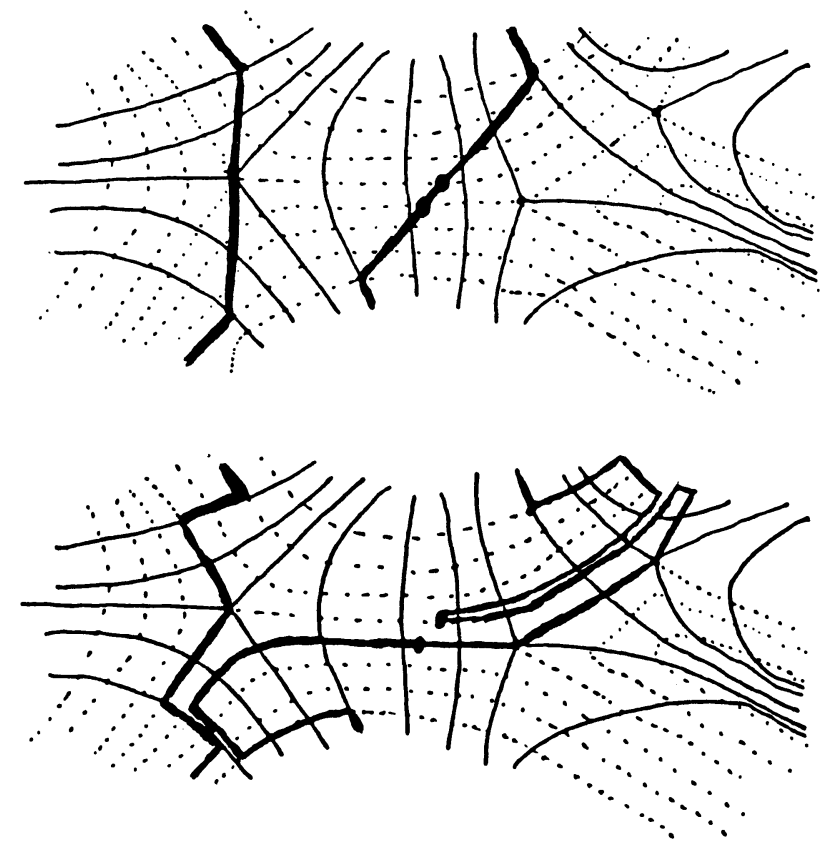

horizontal foliation $=$ solid leaves

FigURE 2

of $\Phi(\rho)$. Let

$$
k_{2}(\rho, \varepsilon([\gamma]))=\max _{p \in M_{\epsilon([\gamma])}(\rho)} \frac{\left\|\partial /\left.\partial x\right|_{p}\right\|_{\rho}}{\left\|\partial /\left.\partial x\right|_{p}\right\|_{4 \Phi(\rho)}}
$$

$k_{2}$ is independent of the $\sigma$-length or orientation of $\{\partial / \partial x\}$, which is why we could be slightly sloppy in defining $\{\partial / \partial x\}$. We next notice that $k_{2}(\rho, \varepsilon([\gamma]))$ is upper semicontinuous as a function of $\rho$ in $T_{g}$. To see this, we first observe that, for any $\delta>0$, if $\rho$ is sufficiently close to $\rho_{0}$ in $T_{g}$, then $M_{\varepsilon([\gamma])}(\rho)$ is contained in a neighborhood of $\sigma$-radius $\delta$ around $M_{\varepsilon([\gamma])}\left(\rho_{0}\right)$. (If not, then there would be a $\delta>0$, a sequence of metrics $\rho_{n} \rightarrow \rho_{0}$, and a sequence of points $x_{n} \in M_{\varepsilon([\gamma])}\left(\rho_{n}\right)$ so that $d_{\sigma}\left(x_{n}, M_{\varepsilon([\gamma])}\left(\rho_{0}\right)\right)>\delta$. But, after possibly passing to a subsequence, we can assume $x_{n} \rightarrow x_{0}$, and since $\Phi(\rho)^{-1}(0)$ varies continuously with $\rho$, we notice that $d\left(x_{n}, \Phi\left(\rho_{n}\right)^{-1}(0) \geq \varepsilon\right.$ implies $d_{\sigma}\left(x_{0}, \Phi\left(\rho_{0}\right)^{-1}(0)\right) \geq \varepsilon$. Therefore, $x_{0} \in M_{\varepsilon([\gamma])}\left(\rho_{0}\right)$ and it could not have been true that $d_{\sigma}\left(x_{n}, M_{\varepsilon([\gamma])}\left(\rho_{0}\right)\right)>\delta$ for every $x_{n}$. Note that this argument hinges on our choice of $M_{\varepsilon([\gamma])}(\rho)$ as being the complement in $M$ of open balls.) 
Thus, for $\rho$ close to $\rho_{0}$, a point $q \in M_{\varepsilon([\gamma])}(\rho)$ at which

$$
\max _{p \in M_{\varepsilon([\gamma])}(\rho)}\left(\left\|\partial /\left.\partial x\right|_{p}\right\|_{\rho} /\left\|\partial /\left.\partial x\right|_{p}\right\|_{4 \Phi(\rho)}\right)
$$

is obtained must lie within a $\delta$-neighborhood of $M_{\varepsilon([\gamma])}\left(\rho_{0}\right)$. So, using that $\left\|\partial /\left.\partial x\right|_{p}\right\|_{\rho} /\left\|\partial /\left.\partial x\right|_{p}\right\|_{4 \Phi(\rho)}$ is continuous both in $p \in M$ and $\rho \in T_{g}$, we find that $k_{2}(\rho, \varepsilon([\gamma]))<k_{2}\left(\rho_{0}, \varepsilon([\gamma])\right)+\xi$, or that $k_{2}$ is upper semicontinuous as a function of $\rho \in T_{g}$.

Let $\Phi_{0} \in \operatorname{SQD}(\sigma)$ and define $\kappa_{r}\left(\Phi_{0}\right)=k_{2}\left(\Phi^{-1}\left(r \Phi_{0}\right), \varepsilon([\gamma])\right) . \quad \kappa_{r}$ is $k_{2}$ restricted to the coordinate sphere of radius $r$ in $\mathrm{QD}(\sigma)$, parametrized by $\operatorname{SQD}(\sigma)$, and $\kappa_{r}\left(\Phi_{0}\right)$ is upper semicontinuous on $\operatorname{SQD}(\sigma)$. Since $\operatorname{SQD}(\sigma)$ is compact, we may define

$$
k_{0}(\|\Phi(\rho)\|,[\gamma])=\max _{\Phi_{0} \in \operatorname{SQD}(\sigma)} \kappa_{\|\Phi(\rho)\|}\left(\Phi_{0}\right) .
$$

Moreover, since $\left(\left\|\partial /\left.\partial x\right|_{p}\right\|_{\Phi^{-1}\left(r \Phi_{0}\right)} /\left\|\partial /\left.\partial x\right|_{p}\right\|_{4 r \Phi_{0}} \downarrow 1\right)$ for fixed $p \in M$ as $r \rightarrow \infty$ by Proposition 4.4(ii), we find that, for fixed $\Phi_{0} \in \operatorname{SQD}(\sigma), \kappa_{r}\left(\Phi_{0}\right) \downarrow 1$ as $r \rightarrow \infty$. Consequently $\kappa_{r}\left(\Phi_{0}\right)$ is a family of upper semicontinuous functions on the compact space $\operatorname{SQD}(\sigma)$, pointwise decreasing monotonically to 1 . So by Dini's Theorem (cf. Royden [16, p. 162]), $\kappa_{r}\left(\Phi_{0}\right)$ converges uniformly to 1.

So $k_{0}(\|\Phi(\rho)\|,[\gamma]) \downarrow 1$ as $\|\Phi(\rho)\| \rightarrow \infty$, the monotonicity coming from the pointwise monotonicity of $\kappa_{\|\Phi(\rho)\|}\left(\Phi_{0}\right)$.

We now show the estimate $k_{0} i(\beta \Phi(\rho),[\gamma])+\eta \geq l_{\rho}([\gamma])$.

Let $\gamma_{h}:[a, b] \rightarrow M$ be a parametrization of a horizontal arc which avoids a neighborhood of $\sigma$-radius $\varepsilon([\gamma])$ of the zeros of $\Phi(\rho)$. Then $\gamma_{h} \subset M_{\varepsilon([\gamma])}(\rho)$ and

$$
\begin{aligned}
l_{\rho}\left(\gamma_{h}\right) & =\int_{a}^{b}\left\|\dot{\gamma}_{h}\right\|_{\rho} d s \\
& =\int_{a}^{b}\left\|\dot{\gamma}_{h}\right\|_{4 \Phi(\rho)} \cdot \frac{\left\|\dot{\gamma}_{h}\right\|_{\rho}}{\left\|\dot{\gamma}_{h}\right\|_{4 \Phi(\rho)}} d s \\
& \leq \sup _{\gamma_{h}} \frac{\left\|\dot{\gamma}_{h}\right\|_{\rho}}{\left\|\dot{\gamma}_{h}\right\|_{4 \Phi(\rho)}} \int_{a}^{b}\left\|\dot{\gamma}_{h}\right\|_{4 \Phi(\rho)} d s \\
& \leq k_{2}(\rho, \varepsilon([\gamma])) i\left(\beta \Phi(\rho), \gamma_{h}\right) \\
& \left.\leq k_{0}(\|\Phi(\rho)\|, \varepsilon(\gamma])\right) i\left(\beta \Phi(\rho), \gamma_{h}\right) .
\end{aligned}
$$

Next let $\gamma_{v}:[a, b] \rightarrow M$ be a parametrization of a vertical arc. Then $i\left(\beta \Phi(\rho), \gamma_{v}\right)=0$; on the other hand, Corollary 4.5 implies that

$$
\left(l_{\Phi^{-1}(t \Phi(\rho) /\|\Phi(\rho)\|)}\left(\gamma_{v}\right)\right) \cdot t^{-1 / 2} \rightarrow 0 \quad \text { as } t \rightarrow \infty .
$$


Because the total $\Phi$-length of vertical arcs of $\gamma$ is bounded by $K([\gamma])$ of which fewer than $L([\gamma])$ subarcs are critical vertical arcs, we recognize that the total $\sigma$-length of the vertical arcs is bounded. We conclude that there is an $\eta(\|\Phi(\rho)\|,[\gamma])$ so that

$$
\sum_{\gamma_{v} \text { vert }} l_{\rho}\left(\gamma_{v}\right)<\eta \text { and } \eta\|\Phi(\rho)\|^{-1 / 2} \rightarrow 0 \quad \text { as }\|\Phi(\rho)\| \rightarrow \infty
$$

Then, letting $\gamma=\gamma_{\Phi(\rho)}$,

$$
\begin{aligned}
l_{\rho}([\gamma]) & \leq l_{\rho}(\gamma)=\sum_{\gamma_{h} \text { hor }} l_{\rho}\left(\gamma_{h}\right)+\sum_{\gamma_{v} \text { vert }} l_{\rho}\left(\gamma_{v}\right) \\
& \leq k_{0} \sum i\left(\beta \Phi(\rho), \gamma_{h}\right)+\eta \\
& =k_{0} i(\beta \Phi(\rho), \gamma)+\eta \\
& =k_{0} i(\beta \Phi(\rho),[\gamma])+\eta
\end{aligned}
$$

with $k_{0}(\|\Phi(\rho)\|,[\gamma]) \downarrow 1$ and $\eta\|\Phi(\rho)\|^{-1 / 2} \rightarrow 0$ as $\|\Phi(\rho)\| \rightarrow \infty$.

We recall the maps $\pi \circ l: T_{g} \rightarrow P\left(\mathbf{R}_{+}^{\mathscr{S}}\right)$ and $\beta: \mathrm{QD}(\sigma) \rightarrow \mathscr{M} \mathscr{F} \subset \mathbf{R}_{+}^{\mathscr{S}}$ that opened $\S \S 4.2$ and 4.3 .

Lemma 4.7. Let $\rho_{n} \rightarrow \partial T_{g}$, i.e., $\rho_{n}$ leaves all compact sets in $T_{g}$. Then $\pi \circ l\left(\rho_{n}\right)$ converges if, and only if, $\pi \circ \beta \Phi\left(\rho_{n}\right)$ converges, and in the case of convergence, the two sequences have the same limit.

Proof. The topology of $T_{g}$ is defined by a finite number of curves $\gamma_{1}, \cdots, \gamma_{k}$ for which, for any $\Phi_{0} \in \operatorname{SQD}(\sigma), \sum_{j} i\left(\beta \Phi_{0},\left[\gamma_{j}\right]\right)>\delta>0$ for some $\delta$. Suppose $\pi \circ l\left(\rho_{n}\right)$ converges; then there exists a sequence of scalars $\lambda_{n}>0$ so that $\lambda_{n} l\left(\rho_{n}\right)$ converges in $\mathbf{R}_{+}^{\mathscr{S}}$ to a nonzero functional in $\mathbf{R}_{+}^{\mathscr{S}}$. In particular, $\lambda_{n}\left(l_{\rho_{n}}\left(\left[\gamma_{1}\right]\right), l_{\rho_{n}}\left(\left[\gamma_{2}\right]\right), \cdots, l_{\rho_{n}}\left(\left[\gamma_{k}\right]\right)\right)$ converges to a nonzero vector in $\mathbf{R}^{k}$, and so

$$
\begin{aligned}
c & >\sum_{j=1}^{k} \lambda_{n} l_{\rho_{n}}\left(\left[\gamma_{j}\right]\right) \\
& >\lambda_{n} \sum_{j=1}^{k} i\left(\beta \Phi\left(\rho_{n}\right),\left[\gamma_{j}\right]\right) \quad \text { by Lemma } 4.6 \\
& =\lambda_{n}\left\|\Phi\left(\rho_{n}\right)\right\|^{1 / 2} \sum_{j=1}^{k} i\left(\beta\left(\Phi\left(\rho_{n}\right) /\left\|\Phi\left(\rho_{n}\right)\right\|,\left[\gamma_{j}\right]\right)\right) \\
& >\left(\lambda_{n}\left\|\Phi\left(\rho_{n}\right)\right\|^{1 / 2}\right) \delta .
\end{aligned}
$$

Thus, $\lambda_{n}=O\left(\left\|\Phi\left(\rho_{n}\right)\right\|^{-1 / 2}\right)$ and so for $[\gamma] \in \mathscr{S}$ and $\eta\left(\left\|\Phi\left(\rho_{n}\right)\right\|,[\gamma]\right)$ as in Lemma 4.6, $\lambda_{n} \eta \rightarrow 0$ as $n \rightarrow \infty$. Then since $k_{0}\left(\left\|\Phi\left(\rho_{n}\right)\right\|,[\gamma]\right) \rightarrow 1$ as $n \rightarrow \infty$, 
and

Lemma 4.6 gives

$$
\begin{aligned}
\lambda_{n} i\left(\beta \Phi\left(\rho_{n}\right),[\gamma]\right) & =\lambda_{n}\left\|\Phi\left(\rho_{n}\right)\right\|^{1 / 2}\left(i\left(\beta\left(\Phi\left(\rho_{n}\right) /\left\|\Phi\left(\rho_{n}\right)\right\|,[\gamma]\right)\right)\right) \\
& <c / \delta \max _{\Phi_{0} \in \operatorname{SQD}(\sigma)} i\left(\beta \Phi_{0},[\gamma]\right),
\end{aligned}
$$

$$
\left|\lambda_{n} l_{\rho_{n}}([\gamma])-\lambda_{n} i\left(\beta \Phi\left(\rho_{n}\right),[\gamma]\right)\right| \rightarrow 0
$$

Thus, $\pi \circ \beta \Phi\left(\rho_{n}\right)$ converges to the same limit as $\pi \circ l\left(p_{n}\right)$. The converse is analogous.

Proof of Theorem 4.1. By dint of Lemma 4.7 we are left with an easy exercise in point-set topology. Define $\psi: T_{g} \cup \mathscr{P} \mathscr{F}=\overline{T_{g}^{t h}} \subset P\left(\mathbf{R}_{+}^{\mathscr{S}}\right) \rightarrow$ $\overline{\mathrm{BQD}(\sigma)}$ by

$$
\psi(x)= \begin{cases}\left(\lim _{n} \frac{\Phi\left(x_{n}\right)}{\left\|\Phi\left(x_{n}\right)\right\|}, 1\right) & \text { if } x \in \partial T_{g} \subset P\left(\mathbf{R}_{+}^{\mathscr{S}}\right) \text { and } x_{n} \rightarrow x \\ \left(\frac{\Phi(x)}{\|\Phi(x)\|}, \frac{4\|\Phi(x)\|}{1+4\|\Phi(x)\|}\right) & \text { if } x \in T_{g}\end{cases}
$$

where we use polar coordinates $(\theta, r)$ for $\operatorname{BQD}(\sigma): \theta \in \operatorname{SQD}(\sigma), r \in[0,1]$.

We first show that $\psi$ is well defined. Suppose $x_{n} \rightarrow x \in \partial T_{g} \subset P\left(\mathbf{R}_{+}^{\mathscr{S}}\right)$ and $x_{n}^{\prime} \rightarrow x$. Then by Lemma $4.7, \lim \pi \circ \beta \Phi\left(X_{n}\right)$ exists and is equal to $\lim \pi \circ \beta \Phi\left(x_{n}^{\prime}\right)$. Furthermore, since $\beta: \mathrm{BQD} \rightarrow \mathscr{M F}$ is a homeomorphism, $\lim _{n} \Phi\left(x_{n}\right) /\left\|\Phi\left(x_{n}\right)\right\|$ exists and equals $\lim _{n} \Phi\left(x_{n}^{\prime}\right) /\left\|\Phi\left(x_{n}^{\prime}\right)\right\|$.

Next we claim that $\psi$ is a homeomorphism onto $\overline{\operatorname{BQD}(\sigma)}$. First we note that $\psi$ is continuous. Suppose $x_{n} \rightarrow x \in \partial T_{g}$; we need to show $\psi\left(x_{n}\right) \rightarrow \psi(x)$. Since $x_{n} \rightarrow \partial T_{g},\left\|\Phi\left(x_{n}\right)\right\| \rightarrow \infty$ which implies $4\left\|\Phi\left(x_{n}\right)\right\| /\left(1+4\left\|\Phi\left(x_{n}\right)\right\|\right) \rightarrow$ 1. So $\psi$ is continuous in its second components; it is continuous in its first components by definition.

The injectivity of $\psi$ on $T_{g}$ follows from Sampson's theorem [17] on the injectivity of $\Phi: T_{g} \rightarrow \mathrm{QD}(\sigma)$. Suppose then that $x, x^{\prime} \in \partial T_{g}$ and $\psi(x)=$ $\psi\left(x^{\prime}\right)$. We can further suppose $x_{n} \rightarrow x \in \partial T_{g}$ and $x_{n}^{\prime} \rightarrow x^{\prime} \in \partial T_{g}$, and so by hypothesis,

$$
\lim _{n} \Phi\left(x_{n}\right) /\left\|\Phi\left(x_{n}\right)\right\|=\lim _{n} \Phi\left(x_{n}^{\prime}\right) /\left\|\Phi\left(x_{n}^{\prime}\right)\right\|
$$

Thus

$$
\lim _{n} \pi \circ \beta \Phi\left(x_{n}\right)=\lim _{n} \pi \circ \beta \Phi\left(x_{n}^{\prime}\right),
$$

and Lemma 4.7 implies that $x=\lim _{n} x_{n}=\lim _{n} x_{n}^{\prime}=x$.

Theorem 3.1 shows that $\psi: T_{g} \rightarrow \mathrm{BQD}$ is surjective. To show that it is surjective as a map $\partial T_{g} \rightarrow \mathrm{SQD}$, we suppose $\theta \in \mathrm{SQD}$ and $r_{n} \theta \rightarrow \theta$. Then $\pi \circ \beta\left(r_{n} \theta\right)$ converges (actually, is constant), and Lemma 4.7 says that $\Phi^{-1}\left(r_{n} \theta\right)$ converges to a point $x \in \partial T_{g}$. Since $\psi$ is continuous,

$$
\psi(x)=\lim _{n} \psi\left(\tilde{\Phi}^{-1} r_{n} \theta\right)=\lim _{n}\left(\theta, r_{n}\right)=(\theta, 1) .
$$

So $\psi$ is surjective. 
Finally, we claim $\psi^{-1}$ is continuous. So we suppose $\left(\theta_{n}, r_{n}\right) \rightarrow(\theta, 1)$, and we show $\psi^{-1}\left(\theta_{n}, r_{n}\right) \rightarrow \psi^{-1}(\theta, 1)$. Since $\left(\theta_{n}, r_{n}\right)$ converges, $\pi \circ \beta\left(r_{n} \theta_{n}\right)$ converges and so, by Lemma 4.7, $\pi \circ \tilde{\Phi}^{-1} r_{n} \theta_{n}=\psi^{-1}\left(\theta_{n}, r_{n}\right)$ converges to $x \in \partial T_{g}$. Since, by definition, $\psi(x)=\lim \left(\theta_{n}, r_{n}\right)=(\theta, 1)$,

$$
\psi^{-1}(\theta, 1)=x=\lim _{n} \psi^{-1}\left(\theta_{n}, r_{n}\right) \text {. }
$$

This concludes the proof of Theorem 4.1.

\subsection{Proof of Proposition 4.3 and Corollary 4.5.}

Proof of Proposition 4.3. Let $M_{\varepsilon}=M \sim \bigcup_{\Phi_{0}(p)=0} B_{\varepsilon}(p)$ where $B_{\varepsilon}(p)$ is a ball of $\sigma$-radius $\varepsilon$ around $p$. Recall that $|\nu(t)|^{2}=\mathscr{L}(t) / \mathscr{H}(t)$ and $0 \leq$ $|\nu(t)|^{2}<1$. Then

$$
\begin{aligned}
\int_{M_{\varepsilon}} & \left(1-\frac{\mathscr{L}}{\mathscr{H}}\right)^{1 / 2} \frac{d A(\sigma)}{A\left(M_{\varepsilon}\right)} \\
& =\int_{M_{\varepsilon}} \frac{(\mathscr{H}-\mathscr{L})^{1 / 2}}{\mathscr{H}^{1 / 2}} \frac{d A(\sigma)}{A\left(M_{\varepsilon}\right)} \\
& \leq\left(\int_{M_{\varepsilon}}(\mathscr{H}-\mathscr{L}) \frac{d A(\sigma)}{A\left(M_{\varepsilon}\right)}\right)^{1 / 2}\left(\int_{M_{\varepsilon}} \frac{1}{\mathscr{H}} \frac{d A(\sigma)}{A\left(M_{\varepsilon}\right)}\right)^{1 / 2} \text { bhere } A\left(M_{\varepsilon}\right)=\text { the } \sigma \text {-area of } M_{\varepsilon} \\
& =\left(\int_{M_{\varepsilon}} \mathscr{H}-\mathscr{L} \frac{d A(\sigma)}{A\left(M_{\varepsilon}\right)}\right)^{1 / 2}\left(\int_{\mathscr{L}^{1 / 2}}^{\mathscr{H}^{1 / 2}} \cdot \frac{1}{\mathscr{H} 1 / 2 \mathscr{L}^{1 / 2}} \frac{d A(\sigma)}{A\left(M_{\varepsilon}\right)}\right)^{1 / 2} \\
& \leq\left(\int_{M_{\varepsilon}} \mathscr{\mathscr { H }}-\mathscr{L} \frac{d A(\sigma)}{A\left(M_{\varepsilon}\right)}\right)^{1 / 2}\left(\int_{M_{\varepsilon}} \frac{\mathscr{L}}{\mathscr{\mathscr { H }}} \frac{d A(\sigma)}{A\left(M_{\varepsilon}\right)}\right)^{1 / 4} \\
& \times\left(\int_{M_{\varepsilon}} \frac{1}{\mathscr{H} \mathscr{L}} \frac{d A(\sigma)}{A\left(M_{\varepsilon}\right)}\right)^{1 / 4} \cdot
\end{aligned}
$$

But $\mathscr{H}-\mathscr{L}>0$, and by Gauss-Bonnet

$$
\int_{M_{\varepsilon}} \mathscr{H}-\mathscr{L} \frac{d A(\sigma)}{A\left(M_{\varepsilon}\right)}<\frac{4 \pi(g-1)}{A\left(M_{\varepsilon}\right)}
$$

however, $\mathscr{H}(t) \mathscr{L}(t)=t^{2}\left|\Phi_{0}\right|^{2} / \sigma^{2}$. So, for all $\varepsilon>0$

$$
\begin{aligned}
\int_{M_{\varepsilon}}\left(1-\frac{\mathscr{L}(t)}{\mathscr{H}(t)}\right)^{1 / 2} d A(\sigma) & \leq \frac{C}{t^{1 / 2}}\left(\int_{M_{\varepsilon}} \frac{\sigma^{2}}{\left|\Phi_{0}\right|^{2}} d A(\sigma)\right)^{1 / 4} \\
& \rightarrow 0 \text { as } t \rightarrow \infty
\end{aligned}
$$

Next we claim that $\left(|\nu(t)|^{2}\right)^{\prime} \geq 0$ for $t$ real and nonnegative, where we use primes to indicate differentiation with respect to $t$. Since $\mathscr{H}(t) \mathscr{L}(t)=$ $t^{2}\left|\Phi_{0}\right|^{2} / \sigma^{2}$ 


$$
\mathscr{H}^{\prime} \mathscr{L}+\mathscr{L}^{\prime} \mathscr{H}=\frac{2 t\left|\Phi_{0}\right|^{2}}{\sigma^{2}}=\frac{2}{t} \mathscr{H} \mathscr{L}, \quad t \neq 0 .
$$

Then, if $\Phi_{0}(p) \neq 0$,

$$
\frac{\mathscr{P}^{\prime}}{\mathscr{H}}(p)+\frac{\mathscr{L}^{\prime}}{\mathscr{L}}(p)=\frac{2}{t}
$$

We first show that $\mathscr{H}^{\prime} \geq 0$. From (IX) we have

$$
t^{2}\left|\Phi_{0}\right|^{2}=\sigma^{2} \mathscr{H}^{2}|\nu(t)|^{2}
$$

so taking a derivative with respect to $t$ gives

$$
0 \leq 2 t\left|\Phi_{0}\right|^{2}=2 \sigma^{2} \mathscr{K} \mathscr{P}^{\prime}|\nu|^{2}+\sigma^{2} \mathscr{H}^{2}\left(|\nu|^{2}\right)^{\prime} .
$$

Sampson [17] and Schoen-Yau [18] both show that $\mathscr{H}>0$. So

$$
\mathscr{H}\left(|\nu|^{2}\right)^{\prime}+\mathscr{H}^{\prime}|\nu|^{2} \geq-\mathscr{H}^{\prime}|\nu|^{2} .
$$

Now suppose $\mathscr{H}^{\prime}<0$ somewhere on $M$. Suppose $p_{0}$ is a minimum of $\mathscr{H}^{\prime} / \mathscr{H}$; naturally $\mathscr{H}^{\prime}\left(p_{0}\right)<0$. Also, since $\Delta \log \mathscr{H}=2 \mathscr{H}\left(1-|\nu|^{2}\right)-2$ by (VI), by taking time derivatives we find that, at the minimum $p_{0}$,

$$
\begin{aligned}
0 & \leq \Delta \mathscr{H}^{\prime} / \mathscr{H}=2 \mathscr{H}^{\prime}\left(1-|\nu|^{2}\right)-2 \mathscr{H}\left(|\nu|^{2}\right)^{\prime} \\
& =2 \mathscr{H}^{\prime}-2\left(\mathscr{H}^{\prime}|\nu|^{2}+\mathscr{H}\left(|\nu|^{2}\right)^{\prime}\right) \\
& \leq 2 \mathscr{H}^{\prime}+2 \mathscr{H}^{\prime}|\nu|^{2} \quad \text { by }(4.4) \\
& <0 \quad \text { since } \mathscr{H}^{\prime}\left(p_{0}\right)<0 \quad \text { by hypothesis. }
\end{aligned}
$$

The contradiction shows that $\mathscr{H}^{\prime} \geq 0$ for all $t$ and at all points on $M$.

We now prove $\left(|\nu(t)|^{2}\right)^{\prime} \geq 0$ by applying the maximum principle to (VI).

Suppose that $p_{1}$ is a maximum of $\mathscr{H}^{\prime} / \mathscr{H}$. Then writing

$$
\Delta \log \mathscr{H}=2 \mathscr{H}-2 \mathscr{L}-2 \text {, }
$$

on differentiating we find, at $p_{1}$

$$
0 \geq \Delta \frac{\mathscr{H}^{\prime}}{\mathscr{H}}=2 \mathscr{H}^{\prime}-2 \mathscr{L}^{\prime}
$$

and so $\mathscr{H}^{\prime}\left(p_{1}\right) \leq \mathscr{L}^{\prime}\left(p_{1}\right)$. This shows that $\Phi\left(p_{1}\right) \neq 0$, as otherwise $\mathscr{L}\left(p_{1}\right)=0$ and

$$
0 \leq \frac{\mathscr{H}^{\prime}\left(p_{1}\right)}{\mathscr{H}\left(p_{1}\right)} \leq \frac{\mathscr{L}^{\prime}\left(p_{1}\right)}{\mathscr{H}\left(p_{1}\right)}=0 .
$$

Thus $\mathscr{H}^{\prime}\left(p_{1}\right) / \mathscr{H}\left(p_{1}\right)=0$, and $\mathscr{H}^{\prime}(p) / \mathscr{H}(p) \equiv 0$ for all $p$ on $M$ at the particular time $t>0$ since $p_{1}$ was a maximum for $\mathscr{H}^{\prime} / \mathscr{H}$. But this is impossible since then

$$
0=\int \mathscr{J}^{\prime}=-\int \mathscr{L}^{\prime}=-\frac{2}{t} \int \mathscr{L}<0
$$

(the last equality by (4.3)). Hence $\Phi\left(p_{1}\right) \neq 0$, and $\Phi_{0}\left(p_{1}\right) \neq 0$. 
(4.3a) now implies that $p_{1}$ is a minimum for $\mathscr{L}^{\prime} / \mathscr{L}$ on $M \sim\left\{\Phi_{0}^{-1}(0)\right\}$. Since $\mathscr{H}-\mathscr{L}=\mathscr{J}>0$ on $M$ by the theorem of Sampson [17] and Schoen-Yau $[18]$, we find that (4.5) gives

$$
\frac{\mathscr{L}^{\prime}(q)}{\mathscr{L}(q)} \geq \frac{\mathscr{L}^{\prime}\left(p_{1}\right)}{\mathscr{L}\left(p_{1}\right)}>\frac{\mathscr{H}^{\prime}\left(p_{1}\right)}{\mathscr{H}\left(p_{1}\right)} \geq \frac{\mathscr{H}^{\prime}(q)}{\mathscr{H}(q)} \text { for } q \in M \sim\left\{\Phi_{0}^{-1}(0)\right\}
$$

so that $\mathscr{L}^{\prime} \mathscr{H}-\mathscr{L}^{\prime}>0$ on $M \sim\left\{\Phi_{0}^{-1}(0)\right\}$. Since $\left(|\nu|^{2}\right)^{\prime}=$ $\left(\mathscr{L}^{\prime} \mathscr{H}-\mathscr{H}^{\prime} \mathscr{L}\right) / \mathscr{H}^{2}$, and $|\nu(p)|^{2}=0$ for all $t$ if $\Phi_{0}(p)=0$, we find that

$$
\left(|\nu(p)|^{2}\right)^{\prime} \geq 0 \text { for all } p \in M \text {. }
$$

Our arguments thus far show that $|\nu(t)(p)|^{2}$ is (by (4.6)) monotone increasing in $t$ and (by (4.2)) converging almost everywhere to 1 to $M \sim\left\{\Phi_{0}^{-1}(0)\right\}$.

Finally, we need to exclude the possibility that $|\nu(t)(p)|^{2} \rightarrow \delta \neq 1$, with $\Phi_{0}(p) \neq 0$. (The author wishes to thank Professor W. Craig for pointing out the following argument.) We consider the function $\log |\nu(t)|^{2}$. Equations (VI) and (VIII) taken together yield, away from the zeros of $\Phi_{0}$,

$$
\Delta \log |\nu|^{2}=\Delta \log \frac{\mathscr{L}}{\mathscr{H}}=-4 \mathscr{J}(t)<0 \quad \text { since } \mathscr{J}(t)>0 .
$$

Also, $\log |\nu(t)|^{2}$ is monotonically increasing on $M_{\varepsilon}$ by (4.6). Since $\log |\nu(t)|^{2}$ is bounded below on $M_{\varepsilon}$, we see from (4.2) that $\log |\nu(t)|^{2} \rightarrow 0$ almost everywhere $(d A(\sigma))$ on $M_{\varepsilon}$. We now represent $(M, \sigma)$ as $\mathbf{H}^{2} / \Gamma$, where $\Gamma$ is a discrete group of isometries of $\mathbf{H}^{2}$; in particular, we consider a fundamental domain $F$ in the unit disk model of $\mathbf{H}^{2}$ for the action of $\Gamma$. Thus we can consider $M_{\varepsilon}$ as a domain $F_{\varepsilon}$ in the disk, so that

$$
\Delta \log |\nu(t)|^{2}<0 \quad \text { on } F_{\varepsilon}
$$

holds with respect to the Euclidean Laplacian. So $\log |\nu(t)|^{2}$ is a monotonically increasing family of superharmonic functions on $F_{\varepsilon}$. A standard theorem (see e.g. Tsuji [25, p. 42, Theorem II.16]) then implies that the limit function $\log |\nu(\infty)|^{2}$ is also superharmonic in the sense of being lower semicontinuous and satisfying

$$
\log |\nu(\infty)|^{2}\left(z_{0}\right) \geq \frac{1}{\pi R^{2}} \iint_{\left|z-z_{0}\right| \leq R} \log |\nu(\infty)|^{2}(z) r d r d \theta
$$

for all $R$ with $\left\{\left|z-z_{0}\right| \leq R\right\} \subset F_{\varepsilon}$ where $z=z_{0}+r e^{i \theta}$. Since $\log |\nu(\infty)|^{2}=0$ almost everywhere (Lebesgue) on $F_{\varepsilon}$, the above inequality shows that $\log |\nu(\infty)|^{2}$ $=0$ everywhere on $F_{\varepsilon}$, and so $|\nu(t)|^{2} \uparrow 1$ everywhere on $M_{\varepsilon}$. Since $\varepsilon$ was arbitrary, we find that $|\nu(t)|^{2} \uparrow 1$ at all points with $\Phi_{0}(p) \neq 0$. 
Proof of Corollary 4.5. (a) Suppose $\|\partial / \partial y\|_{\rho_{t}}>\delta>0$ on a set $A$ of positive measure with $p \in A$. Then on $A$,

$$
\mathscr{J}(w(t))=\|\partial / \partial x\|_{\rho_{t}} \cdot\|\partial / \partial y\|_{\rho_{t}}>\|\partial / \partial x\|_{\rho_{t}} \delta
$$

Since $\|\partial / \partial x\|_{\rho_{t}} \geq 2 t\left|\Phi_{0}\right|^{1 / 2} \rightarrow \infty$, we have $\int_{A} \mathcal{J}(w(t)) d A(\sigma) \rightarrow \infty$, which is impossible.

(b) We recall that $\partial / \partial x$ is tangent to the horizontal (maximal stretch) foliation, and $\|\partial / \partial x\|_{\sigma}=1$. Then

$$
\begin{aligned}
\frac{\|\partial / \partial x\|_{\rho_{t}}}{t^{1 / 2}} & =\frac{\mathscr{H}(t)^{1 / 2}+\mathscr{L}(t)^{1 / 2}}{t^{1 / 2}} \\
& =\left(1+\left(\frac{\mathscr{L}(t)}{\mathscr{H}(t)}\right)^{1 / 2}\right) \frac{\mathscr{H}(t)^{1 / 2}}{t^{1 / 2}} \\
& =(1+|\nu(t)|) \frac{\left|\Phi_{0}\right|^{1 / 2}}{\sigma^{1 / 2}} \frac{1}{|\nu(t)|^{1 / 2}} \\
& \left(\text { since, by }(\mathrm{IX}), t^{1 / 2}\left|\Phi_{0}\right|^{1 / 2}=\sigma^{1 / 2} \mathscr{H}(t)^{1 / 2}|\nu(t)|^{1 / 2}\right) \\
& =(1+|\nu(t)|) \mathscr{H}(1)^{1 / 2}\left(\frac{|\nu(1)|}{|\nu(t)|}\right)^{1 / 2},
\end{aligned}
$$

since, also by (IX), $\left|\Phi_{0}\right|^{1 / 2}=\sigma^{1 / 2} \mathscr{H}(1)^{1 / 2}|\nu(1)|^{1 / 2}$.

We now want to show that (4.8) is bounded above and below. To this end, observe that $1 \leq 1+|\nu(t)| \leq 2$, and that $0<c_{2} \leq \mathscr{H}(1)^{1 / 2} \leq c_{3}$ since $M$ is compact and $\mathscr{H}(1)>0$ ([17], [18] again). For the final expression in (4.8), we notice that since $\gamma$ does not contain a zero of $\Phi_{0}$, it does not contain a zero of $\nu(1)=\Phi_{0} /(\sigma \mathscr{H}(1))$. Since $|\nu(t)|$ is increasing by (4.6), for $t>1$ we have

$$
0<\min _{\gamma}|\nu(1)|^{1 / 2}<\left(\frac{|\nu(1)|}{|\nu(t)|}\right)^{1 / 2}<1,
$$

and the statement follows from $l_{\rho_{t}}(\gamma)=\int_{\gamma}\|\partial / \partial x\|_{\rho_{t}} d s_{\sigma}$.

(c) We cannot define a $\sigma$-orthonormal frame field $\partial / \partial x, \partial / \partial y$ at a zero of $\Phi_{0}$. However,

$$
\|\partial / \partial y\|_{\rho_{t}}=\mathscr{H}(t)^{1 / 2}-\mathscr{L}(t)^{1 / 2}
$$

away from a zero of $\Phi_{0}$, and

$$
\begin{aligned}
\left(\mathscr{H}(t)^{1 / 2}-\mathscr{L}(t)^{1 / 2}\right) t^{-1 / 2} & =(1-|\nu(t)|) \mathscr{H}(1)^{1 / 2}\left(\frac{|\nu(1)|}{|\nu(t)|}\right)^{1 / 2} \\
& <(1-|\nu(t)|) \mathscr{H}(1)^{1 / 2}
\end{aligned}
$$


away from the zeros of $\Phi_{0}$ by (4.7). Since both sides are continuous at the zeros of $\Phi_{0}$, the inequality holds everywhere, and so

$$
\begin{aligned}
l_{\rho_{t}}(\gamma) t^{-1 / 2} & =t^{-1 / 2} \int_{\gamma} \mathscr{H}(t)^{1 / 2}-\mathscr{L}(t)^{1 / 2} d s_{\sigma} \\
& <\int_{\gamma}(1-|\nu(t)|) \mathscr{H}(1)^{1 / 2} d s_{\sigma} .
\end{aligned}
$$

Since $|\nu(t)| \geq 0$, the right-hand side converges to zero by Proposition 4.3 and the Dominated Convergence Theorem.

\section{Local variational formulas}

5.1. A computational method. Choose a complex basis $\Phi_{1}, \cdots$, $\Phi_{3 g-3}$ for QD $(\sigma)$; then we can consider the associated local coordinates for $T_{g}$. Specifically, for $t=\left(t_{1}, \cdots, t_{3 g-3}\right) \in \mathbf{C}^{3 g-3}$, define $\Phi(t)=\sum t_{j} \Phi_{j}$ and consider the metric

$$
\rho(t)=\Phi(t) d z^{2}+\sigma e(t) d z d z+\Phi(t) d z^{2} .
$$

The identity map id: $(M, \sigma) \rightarrow(M, \rho(t))$ is harmonic; in this section, we investigate how geometric quantities associated with a harmonic map vary as functions of $t$, for small $t$. Our approach will be first to determine the variations of $\mathscr{H}(t)$ and $\mathscr{L}(t)$ for small $t$, and then to apply those formulas to equations (I), (II) and (IV) to derive the variations $\mathscr{J}(t), \nu(t)$ of $e(t)$. The computations are straightforward after a single observation.

Lemma 5.1. $\mathscr{H}(t) \geq 1 . \mathscr{H}(t) \equiv 1 \Leftrightarrow t=0 \Leftrightarrow \mathscr{L}(t) \equiv 0$.

Proof. As we noted earlier, Sampson [17] and Schoen-Yau [18] both proved $\mathscr{H}(t)>0$. So $\log \mathscr{H}(t)$ is a well-defined function on all of $M$. Suppose $\mathscr{H}(t)$ has a minimum at $p$. Then

$$
0 \leq \Delta \log \mathscr{H}=2 \mathscr{H}-2 \mathscr{L}-2
$$

Since $\mathscr{L} \geq 0$,

$$
\mathscr{H}(t)(p) \geq \mathscr{L}+1 \geq 1
$$

But $p$ is a minimum for $\mathscr{H}$, so we see $\mathscr{H}(t)(q) \geq 1$ for all $q \in M$. Also, if $\mathscr{H} \equiv 1$, then $0=\Delta \log \mathscr{H}=2(1)-2 \mathscr{L}-2$, and so $\mathscr{L} \equiv 0$. Hence the map is conformal and $\rho(t)=\sigma$. q.e.d.

The rest of this section is devoted to computations of the $t$-derivative of various quantities associated with the harmonic maps. (We emphasize that in contrast to the previous section, $t$ is now a complex variable.) We postpone discussing the $t$-smoothness of these quantities until after Corollary 5.4.

Corollary 5.2. (i) $\partial /\left.\partial t^{\alpha}\right|_{0} \mathscr{H}(t)=0$. 
(ii) $\partial /\left.\partial t^{\alpha}\right|_{0} \mathscr{L}(t)=0$.

(iii) $\partial /\left.\partial t^{\alpha}\right|_{0} \nu(t)=\bar{\Phi}_{\alpha} / \sigma$.

(iv) $\partial /\left.\partial t^{\alpha}\right|_{0} \mathscr{J}(t)=0$ (Ahlfors' lemma; see [2, Lemma 2]).

(v) $\partial /\left.\partial t^{\alpha}\right|_{0} e(t)=0 ; \partial /\left.\partial t^{\alpha}\right|_{0} E(\rho(t))=0$.

Proof. (i) $\mathscr{H}(0) \equiv 1, \mathscr{H}(t) \geq 1$.

(ii) $\mathscr{L}(0) \equiv 0, \mathscr{L}(t) \geq 0$.

(iii) $\Phi(t)=\sigma \mathscr{H}(t) \bar{\nu}(t)$. Applying $\partial /\left.\partial t^{\alpha}\right|_{0}$ to each side,

$$
\begin{aligned}
\Phi_{\alpha} & =\left.\sigma \frac{\partial}{\partial t^{\alpha}}\right|_{0} \mathscr{H}(t) \bar{\nu}(0)+\left.\sigma \mathscr{H}(0) \frac{\partial}{\partial t^{\alpha}}\right|_{0} \bar{\nu}(t) \\
& =\left.\sigma \frac{\partial}{\partial t^{\alpha}}\right|_{0} \bar{\nu}(t) \quad \text { by (i). }
\end{aligned}
$$

(iv) $\mathscr{J}(t)=\mathscr{H}(t)-\mathscr{L}(t)$; (iii) shows this to be equivalent to Ahlfors' lemma.

(v) $e(t)=\mathscr{H}(t)+\mathscr{L}(t) ; E(\rho(t))=\int e(\rho(t))$.

Let $\operatorname{Sym}(0,2)$ denote the space of symmetric $(0,2)$ tensors on $M$, and $\mathscr{M}$ the space of positive definite symmetric $(0,2)$ tensors. Now, forgetting the complex structure on $T_{g}$, the family $\rho(t)$ is a $6 g-g$ (real) dimensional subspace of $\mathscr{M} \subset \operatorname{Sym}(0,2)$ with coordinates coming from the real basis $\left\{\Phi_{j}, i \Phi_{j}\right\}$ of $\mathrm{QD}(\sigma)$. Since $\mathscr{M}$ is open in $\operatorname{Sym}(0,2)$, we can identify $T_{\sigma} \mathscr{M}$ with $\operatorname{Sym}(0,2)$; then, using Corollary $5.2(\mathrm{v})$, the tangent vector to the one real parameter family $\rho(t)=t \Phi_{0} d z^{2}+\sigma e(t) d z d \bar{z}+t \bar{\Phi}_{0} d z^{2}$ is given by $\Phi_{0} d z^{2}+\bar{\Phi}_{0} d \bar{z}^{2}=$ $2 \operatorname{Re}\left\{\Phi_{0} d z^{2}\right\} \in \operatorname{Sym}(0,2)$. Thus we identify $T_{\sigma} T_{g} \approx \mathrm{QD}(\sigma)$. All of this is developed slightly differently in Fischer-Tromba [6]; they go on in [7] to show that the natural $L^{2}$ inner product on $T_{\sigma} \mathscr{M}$ descends to a metric on $T_{g}$ given by

$$
\left\langle\Phi_{1}, \Phi_{2}\right\rangle=2 \operatorname{Re} \int \frac{\Phi_{1} \overline{\Phi_{2}}}{\sigma^{2}} d A(\sigma)
$$

This metric is called the Weil-Petersson metric for $T_{g}$; its hermitian form is

$$
\left\langle\Phi_{1}, \Phi_{2}\right\rangle_{\mathrm{wp}}=\int \frac{\Phi_{1} \overline{\Phi_{2}}}{\sigma^{2}} d A(\sigma)
$$

The metric is known to have negative sectional curvature, not to be complete but to be geodesically convex (see [27], [26], [28]).

We now continue our computations. 


\section{Proposition 5.3.}

(i)

$$
\begin{aligned}
& \left.\frac{\partial^{2}}{\partial t^{\alpha} \partial t^{\bar{\beta}}}\right|_{0} \mathscr{L}(t)=\frac{\Phi_{\alpha} \bar{\Phi}_{\beta}}{\sigma^{2}} \\
& \left.\frac{\partial^{2}}{\partial t^{\alpha} \partial t^{\beta}}\right|_{0} \mathscr{L}(t)=0=\left.\frac{\partial^{2}}{\partial t^{\bar{\alpha}} \partial t^{\bar{\beta}}}\right|_{0} \mathscr{L}(t) \\
& \left.\frac{\partial^{2}}{\partial t^{\alpha} \partial t^{\bar{\beta}}}\right|_{0} \mathscr{H}(t)=-2(\Delta-2)^{-1} \frac{\Phi_{\alpha} \bar{\Phi}_{\beta}}{\sigma^{2}} \\
& \left.\frac{\partial^{2}}{\partial t^{\alpha} \partial t^{\beta}}\right|_{0} \mathscr{H}(t)=0=\left.\frac{\partial^{2}}{\overline{\partial t^{\alpha}} \partial t^{\beta}}\right|_{0} \mathscr{H}(t) .
\end{aligned}
$$

Proof. Our method is to take $t$-derivatives of equation (III) to determine the $t$ variations of $\mathscr{L}(t)$; then we use that information to solve for the $t$ variations of $\mathscr{H}(t)$ in the $t$-derivatives of equation (VI). This method is in fact quite general: we discuss it more after Corollary 5.4.

For convenience set $\Phi(t)=t^{\alpha} \Phi_{\alpha}+t^{\beta} \Phi_{\beta}$. Then equation (III) can be written as

$$
\sigma^{2} \mathscr{H}(t) \mathscr{L}(t)=|\Phi(t)|^{2}=\left|t^{\alpha}\right|^{2}\left|\Phi_{\alpha}\right|^{2}+t^{\alpha} t^{\bar{\beta}} \Phi_{\alpha} \bar{\Phi}_{\beta}+\overline{t^{\alpha}} t^{\beta} \bar{\Phi}_{\alpha} \Phi_{\beta}+\left|t^{\beta}\right|^{2}\left|\Phi_{\beta}\right|^{2} .
$$

Applying $\partial^{2} /\left.\partial t^{\alpha} \partial t^{\bar{\beta}}\right|_{0}$ to both sides gives

$$
\begin{aligned}
\sigma^{2}\left[\mathscr{L}(0) \frac{\partial^{2}}{\partial t^{\alpha} \partial t^{\bar{\beta}}}\left|\mathscr{H}(t)+\frac{\partial}{\partial t^{\alpha}}\right| \mathscr{H}(t) \frac{\partial}{\partial t^{\bar{\beta}}} \mid \mathscr{L}(t)\right. \\
\left.+\frac{\partial}{\partial t^{\bar{\beta}}}\left|\mathscr{H}(t) \frac{\partial}{\partial t^{\alpha}}\right| \mathscr{L}(t)+\mathscr{H}(0) \frac{\partial^{2}}{\partial t^{\alpha} \partial t^{\bar{\beta}}} \mathscr{L}(t)\right]=\Phi_{\alpha} \overline{\Phi_{\beta}} .
\end{aligned}
$$

Corollary 5.2(ii) and Lemma 5.1 reduce this to the first part of (i). The other part of (i) is analogous.

Now apply $\partial^{2} /\left(\partial t^{\alpha} \overline{\partial t^{\beta}}\right)$ to (VI) giving, after changing the order of differentiation,

$$
\begin{aligned}
& \Delta\left(\left.\mathscr{H}(0) \frac{\partial^{2}}{\partial t^{\alpha} \partial t^{\bar{\beta}}}\right|_{0} \mathscr{H}(t)-\left.\left.\frac{\partial}{\partial t^{\alpha}}\right|_{0} \mathscr{H}(t) \frac{\partial}{\partial t^{\bar{\beta}}}\right|_{0} \mathscr{H}(t)\right) / \mathscr{H}(0)^{2} \\
& =\left.2 \frac{\partial^{2}}{\partial t^{\alpha} \partial t^{\bar{\beta}}}\right|_{0} \mathscr{H}(t)-\left.2 \frac{\partial^{2}}{\partial t^{\alpha} \partial t^{\bar{\beta}}}\right|_{0} \mathscr{L}(t)
\end{aligned}
$$

Corollary 5.2(ii), Lemma 5.1, and the above computation of $\left(\partial^{2} / \partial t^{\alpha} \partial t^{\bar{\beta}}\right) \mathscr{L}(t)$ give

$$
\left.\Delta \frac{\partial^{2}}{\partial t^{\alpha} \partial t^{\bar{\beta}}}\right|_{0} \mathscr{H}(t)=\left.2 \frac{\partial^{2}}{\partial t^{\alpha} \partial t^{\bar{\beta}}}\right|_{0} \mathscr{H}(t)-2 \frac{\Phi_{\alpha} \overline{\Phi_{\beta}}}{\sigma^{2}}
$$

which yields (ii). 
Corollary 5.4. (i) $\partial^{2} /\left.\partial t^{\alpha} \overline{\partial t^{\beta}}\right|_{0} \nu(t)=0$; consequently, the section $\nu(t)$ : $T_{g} \rightarrow \operatorname{Belt}(\sigma)$ is Weil-Petersson geodesic at $t=0$, where $\operatorname{Belt}(\sigma)$ is the unit ball of Beltrami differentials on $(M, \sigma)$.

(ii) (Wolpert [26], Royden [15])

$$
\left.\frac{\partial^{2}}{\partial t^{\alpha} \partial t^{\bar{\beta}}}\right|_{0} \mathscr{J}(t)=-2(\Delta-2)^{-1} \frac{\Phi_{\alpha} \overline{\Phi_{\beta}}}{\sigma^{2}}-\frac{\Phi_{\alpha} \overline{\Phi_{\beta}}}{\sigma^{2}} .
$$

(iii)

$$
\left.\frac{\partial^{2}}{\partial t^{\alpha} \overline{\partial t^{\beta}}}\right|_{0} e(t)=-2(\Delta-2)^{-1} \frac{\Phi_{\alpha} \overline{\Phi_{\beta}}}{\sigma^{2}}+\frac{\Phi_{\alpha} \overline{\Phi_{\beta}}}{\sigma^{2}} .
$$

(We note that the condition of being Weil-Petersson geodesic at a point of $T_{g}$ requires only agreement through second order with a Weil-Petersson geodesic through that point.)

Proof. Similar to Corollary $1.2 ; \nu(t)$ is Weil-Petersson geodesic since, by Ahlfors [2], $\mu(t)=t((\bar{\Phi}(\alpha) / \sigma)$ is Weil-Petersson geodesic at $t=0$, and so agrees with $\nu(t)$ through second order.

Remarks. (i) Royden [15] and Wolpert [27] derive the second variation of hyperbolic area density along a Weil-Petersson geodesic (ii) with completely different methods; that computation is the linchpin in their derivation of the curvature tensor for the Weil-Petersson metric. We note that $e(t)$ now has a double role: it is both the energy density of the harmonic map $w:(M, \sigma) \rightarrow$ $(M, \rho(t))$, and it is the $(1,1)$ part of the hyperbolic metric whose form in a coordinate neighborhood is

$$
\rho(t)=\Phi(t) d z^{2}+\sigma e(t) d z d \bar{z}+\overline{\Phi(t)} d \bar{z}^{2} .
$$

Since the slice $\rho(t)$ is Weil-Petersson geodesic at $t=0$, the formulas of Wolpert and Royden, together with the Ahlfors lemma, would have been enough to prove (iii) in the second interpretation of $e(t)$.

(ii) If we know the first $n$ derivatives of $\mathscr{H}(t)$ and $\mathscr{L}(t)$, then by taking the $(n+1)$ st derivative of equation (III), we can derive the $(n+1)$ st derivative of $\mathscr{L}(t)$. If we know the first $n$ derivatives of $\mathscr{H}(t)$ and the first $n+1$ derivatives of $\mathscr{L}(t)$, we can derive the $(n+1)$ st derivative of $\mathscr{H}(t)$ by taking the $(n+1)$ st derivative of equation (VI). Thus we can recursively compute all of the derivatives of $\mathscr{H}(t)$ and $\mathscr{L}(t)$, and hence compute an explicit formal power series expansion for $\rho(t)$ (using (5.3)). In a forthcoming paper, we will show that this series converges for small $|t|$.

(iii) We can continue this program to find that all of the odd derivatives of $\mathscr{H}(t)$ and $\mathscr{L}(t)$ vanish. We expect this since $\mathscr{H}(t)$ and $\mathscr{L}(t)$ are solutions 
of the equations $\Delta \log \mathscr{H}(t)=2 \mathscr{H}(t)-2|\Phi(t)|^{2} /\left(\sigma^{2} \mathscr{H}(t)\right)-2$ and $\mathscr{L}(t)=$ $|\Phi(t)|^{2} /\left(\sigma^{2} \mathscr{K}(t)\right)$, both of which depend only on the modulus of $\Phi(t)$ and not on its argument.

(iv) We can compute the formal power series expansion in $t$ of $\nu(t)$ to any order, say $k$. Corresponding to such an expansion is a $C^{k}$ variation in hyperbolic metrics (Ahlfors-Bers [2]), and hence a $C^{k}$ variation in the harmonic maps by a theorem of Sampson [17]. Consequently, all of the quantities associated to the harmonic maps are $C^{\infty}$ in $t$.

(v) In a remark at the end of $\S 3$, we noted that we could pursue a study of $T_{g}$ through equations (III) and (VI), using $\mathrm{QD}(\sigma)$ (instead of the Beltrami differentials, $\operatorname{Belt}(\sigma)$, on $(M, \sigma))$ as a parameter space. Both $\mathrm{QD}(\sigma)$ and $\operatorname{Belt}(\sigma)$ are linear spaces, but we do not expect the rays to correspond. From Corollary 5.4(i) and Remark (ii) above, we see that the rays agree through second order, but no further.

Definition 5.5. Set $D=-2(\Delta-2)^{-1}$ where $\Delta$ is the Laplace-Beltrami operator on $(M, \sigma)$.

Then $D$ is a self-adjoint, compact operator which is the identity on the constant functions.

The technique of (ii) yields

\section{Proposition 5.6.}

$$
\begin{aligned}
& \left.\frac{\partial^{4}}{\partial t^{\alpha} \partial t^{\bar{\beta}} \partial t^{\gamma} \partial t^{\bar{\delta}}}\right|_{0} \mathscr{L}(t) \\
& =-\left[D\left(\frac{\Phi_{\alpha} \bar{\Phi}_{\beta}}{\sigma^{2}}\right) \frac{\Phi_{\gamma} \Phi_{\delta}}{\sigma^{2}}+D\left(\frac{\Phi_{\gamma} \bar{\Phi}_{\delta}}{\sigma^{2}}\right) \frac{\Phi_{\alpha} \bar{\Phi}_{\beta}}{\sigma^{2}}\right. \\
& \left.\quad+D\left(\frac{\bar{\Phi}_{\beta} \Phi_{\gamma}}{\sigma^{2}}\right)\left(\frac{\Phi_{\alpha} \bar{\Phi}_{\delta}}{\sigma^{2}}\right)+D\left(\frac{\Phi_{\alpha} \bar{\Phi}_{\delta}}{\sigma^{2}}\right) \frac{\bar{\Phi}_{\beta} \Phi_{\gamma}}{\sigma^{2}}\right] ;
\end{aligned}
$$

(ii)

$$
\begin{aligned}
\left.\frac{\partial^{4}}{\partial t^{\alpha} \partial t^{\beta} \partial t^{\gamma} \partial t^{\delta}}\right|_{0} \mathscr{L}(t) & =\left.\frac{\partial^{4}}{\partial t^{\bar{\alpha}} \partial t^{\beta} \partial t^{\gamma} \partial t^{\delta}}\right|_{0} \mathscr{L}(t) \\
& =\left.\frac{\partial^{4}}{\partial t^{\bar{\alpha}} \partial t^{\bar{\beta}} \partial t^{\bar{\gamma}} \partial t^{\delta}}\right|_{0} \mathscr{L}(t) \\
& =\left.\frac{\partial^{4}}{\partial t^{\bar{\alpha}} \partial t^{\bar{\beta}} \partial t^{\bar{\gamma}} \partial t^{\bar{\delta}}}\right|_{0} \mathscr{L}(t)=0 .
\end{aligned}
$$

5.2. The geometry of the energy function. Next we interpret these formulas in terms of the geometry of Teichmüller space. We can consider the total energy of the harmonic map from $(M, \sigma)$ to $(M, \rho(t))$ as a function $E(\sigma ; \rho(t))$ on $T_{g}$. It is not hard to show that $E(\sigma ; \rho(t))$ has a global minimum at $t=0$ (see [11]). Since $t=0$ is a critical point of $E(\sigma, \rho(t))$ (also shown by 
the second part of Corollary 5.2(v)), the Hessian of $E(\sigma ; \rho(t))$ is a coordinateindependent inner product on $T_{\sigma} T_{g}$. So, using the properties of $D$, we find that Corollary 5.4(iii) implies

Theorem 5.7. Hess $\left.E(\sigma ; \rho(t))\right|_{0}\left(\Phi_{\alpha}, \Phi_{\beta}\right)=2\left\langle\Phi_{\alpha}, \Phi_{\beta}\right\rangle_{\mathrm{wp}}$.

Closely allied to $E(\sigma ; \rho(t))$ is the function $E(\rho(t) ; \sigma)$, the total energy of the harmonic map from the variable source $(M, \rho(t))$ to the fixed target $(M, \sigma)$. Tromba [23] has proved a result analogous to Theorem 5.7 for this function which we now show follows formally from Corollary 5.2.

Corollary 5.8 (Tromba [23]). Hess $\left.E(\rho(t) ; \sigma)\right|_{0}\left(\Phi_{\alpha}, \Phi_{\beta}\right)=2\left\langle\Phi_{\alpha}, \Phi_{\beta}\right\rangle_{\mathrm{wp}}$.

Proof. Let $\rho\left(t^{\alpha}, t^{\beta}\right)=\Phi^{-1}\left(t^{\alpha} \Phi_{\alpha}+t^{\beta} \Phi_{\beta}\right)$ and $\rho\left(t^{\alpha}\right)=\Phi^{-1}\left(t^{\alpha} \Phi_{\alpha}\right)$; also let $\mathscr{L}\left(\rho_{1} ; \rho_{2}\right)$ be the anti-holomorphic energy density function (on $M$ ) of the harmonic map from $\left(M, \rho_{1}\right)$ to $\left(M, \rho_{2}\right)$. Then we have

$$
0=\left.\frac{\partial}{\partial t^{\alpha}}\right|_{t^{\alpha}=0} \mathscr{L}\left(\rho\left(t^{\beta}\right) ; \rho\left(t^{\alpha}, t^{\beta}\right)\right)
$$

because $\mathscr{L}\left(\rho\left(t^{\beta}\right), \rho\left(t^{\alpha}, t^{\beta}\right)\right) \geq 0$ with equality only when $t_{\alpha}=0$. Thus

$$
\begin{aligned}
0 & =\left.\left.\frac{\partial}{\partial t^{\bar{\beta}}}\right|_{t^{\beta}=0} \frac{\partial}{\partial t^{\alpha}}\right|_{t^{\alpha}=0} \mathscr{L}\left(\rho\left(t^{\beta}\right) ; \rho\left(t^{\alpha}, t^{\beta}\right)\right) \\
& =\left.\frac{\partial}{\partial t^{\alpha} \partial t^{\bar{\beta}}}\right|_{t=0} \mathscr{L}\left(\rho(0) ; \rho\left(t^{\alpha}, t^{\beta}\right)\right)+\left.\frac{\partial^{2}}{\partial t^{\alpha} \partial t^{\bar{\beta}}}\right|_{t=0} \mathscr{L}\left(\rho\left(t^{\alpha}\right) ; \rho\left(t^{\beta}\right)\right) .
\end{aligned}
$$

Similarly

$$
\begin{aligned}
0 & =\left.\frac{\partial^{2}}{\partial t^{\bar{\beta}} \partial t^{\alpha}}\right|_{t=0} \mathscr{L}\left(\rho\left(t^{\alpha}, t^{\beta}\right) ; \rho\left(t^{\beta}\right)\right) \\
& =\left.\frac{\partial^{2}}{\partial t^{\alpha} \partial^{\bar{\beta}}}\right|_{t=0} \mathscr{L}\left(\rho\left(t^{\alpha}, t^{\beta}\right) ; \rho(0)\right)+\left.\frac{\partial^{2}}{\partial t^{\alpha} \partial t^{\bar{\beta}}}\right|_{t=0} \mathscr{L}\left(\rho\left(t^{\alpha}\right) ; \rho\left(t^{\beta}\right)\right) .
\end{aligned}
$$

So

$$
\left.\frac{\partial^{2}}{\partial t^{\alpha} \partial t^{\bar{\beta}}}\right|_{t=0} \mathscr{L}\left(\rho\left(t^{\alpha}, t^{\beta}\right) ; \sigma\right)=\left.\frac{\partial^{2}}{\partial t^{\alpha} \partial t^{\bar{\beta}}}\right|_{t=0} \mathscr{L}\left(\rho\left(t^{\alpha}, t^{\beta}\right)\right)=\frac{\Phi_{\alpha} \bar{\Phi}_{\beta}}{\sigma^{2}} .
$$

Since the same technique works for $\mathscr{H}$, the theorem follows immediately from $e=\mathscr{H}+\mathscr{L}$.

Royden [15], Tromba [24], and Wolpert [27] have recently computed the curvature tensor for the Weil-Petersson metric in terms of the operator $D$.

Theorem 5.9 (Royden, Tromba, Wolpert).

$$
R_{\alpha \bar{\beta} \gamma \bar{\delta}}=\int_{M}\left(D \frac{\Phi_{\alpha} \bar{\Phi}_{\beta}}{\sigma^{2}}\right) \frac{\Phi_{\gamma} \bar{\Phi}_{\delta}}{\sigma^{2}}+\left(D \frac{\Phi_{\alpha} \bar{\Phi}_{\delta}}{\sigma^{2}}\right)\left(\frac{\bar{\Phi}_{\beta} \Phi_{\gamma}}{\sigma^{2}}\right) d A(\sigma) .
$$

By coincidence of formulas we find 


\section{Proposition 5.10.}

$$
\frac{\partial^{4}}{\partial t^{\alpha} \partial t^{\bar{\beta}} \partial t^{\gamma} \partial t^{\bar{\delta}}} \int_{t=0} E(\sigma, \rho(t))=-4 R_{\alpha \bar{\beta} \gamma \bar{\delta}}(\sigma) .
$$

Proof.

$$
\begin{aligned}
E(\sigma ; \rho(t))-4 \pi(g-1) & =\int e(\sigma, \rho(t)) d A(\sigma)-\int \mathscr{J}(\sigma ; \rho(t)) d A(\sigma) \\
& =2 \int \mathscr{L}(\sigma ; \rho(t)) d A(\sigma) .
\end{aligned}
$$

So

$$
\left.\frac{\partial^{4}}{\partial t^{\alpha} \partial t^{\bar{\beta}} \partial t^{\gamma} \partial t^{\bar{\delta}}}\right|_{0} E(\sigma ; \rho(t))=2 \int \frac{\partial^{4}}{\partial t^{\alpha} \partial t^{\bar{\beta}} \partial t^{\gamma} \partial t^{\bar{\delta}}} \mathscr{L}(\sigma ; \rho(t)) d A(\sigma) .
$$

Remark. Because our coordinate system is geodesic at $t=0$, and the Weil-Petersson metric at $\rho\left(t^{\alpha}, t^{\beta}\right)$ is given by

$$
\left.\frac{1}{2} \frac{\partial^{2}}{\partial t^{\gamma} \partial t^{\bar{\delta}}}\right|_{t^{\alpha}=t^{\delta}=0} E\left(\rho\left(t^{\alpha}, t^{\beta}\right) ; \rho\left(t^{\alpha}, t^{\beta}, t^{\gamma}, t^{\delta}\right)\right),
$$

we know that

$$
R_{\alpha \bar{\beta} \gamma \bar{\delta}}(\sigma)=\left.\frac{1}{2} \frac{\partial^{4}}{\partial t^{\alpha} \partial t^{\bar{\beta}} \partial t^{\gamma} \partial t^{\bar{\delta}}}\right|_{0} E\left(\rho\left(t^{\alpha}, t^{\beta}\right) ; \rho\left(t^{\alpha}, t^{\beta}, t^{\gamma}, t^{\delta}\right)\right) .
$$

Thus, Proposition 5.10, since it does not involve a variation of the source metric, is somewhat surprising. Recently, Jost [12] has used the above methods to compute $R_{\alpha \bar{\beta} \gamma \bar{\delta}}(\sigma)$ via the energy of harmonic maps.

\section{References}

[1] L. Ahlfors, Some remarks on Teichmüller's space of Riemann surfaces, Ann. of Math. (2) 74 (1961) 171-191.

[2] L. Ahlfors \& L. Bers, Riemann's mapping theorem for variable metrics, Ann. of Math. (2) 72 (1960) 385-404.

[3] C. J. Earle \& J. Eells, A fibre bundle description of Teichmüller theory, J. Differential Geometry 3 (1969) 19-43.

[4] J. Eells \& J. H. Sampson, Harmonic mappings of Riemannian manifolds, Amer. J. Math. 86(1964) 109-160.

[5] A. Fathi, F. Laudenbach \& V. Poenaru, Traveaux de Thurston sur les surfaces, Asterisque 66-66 (1979).

[6] A. E. Fischer \& A. J. Tromba, On a purely Riemannian proof of the structure and dimension of the unramified moduli space of a compact Riemann surface, Math. Ann. 267 (1984) 311-345.

[7] _ On the Weil-Petersson metric on Teichmüller space, Trans. Amer. Math. Soc. 284 (1984) 319-335.

[8] M. Gerstenhaber \& H. E. Rauch, On extremal quasi-conformal mappings. I, II, Proc. Nat. Acad. Sci. U.S.A. 40 (1954) 808-812, 991-994.

[9] P. Hartman, On homotopic harmonic maps, Canad. J. Math. 19 (1967) 673-687. 
[10] J. Hubbard \& H. Masur, Quadratic differentials and foliations, Acta. Math. 142 (1979) 221-274.

[11] J. Jost, Harmonic maps between surfaces, Lecture Notes in Math., Vol. 1062, Springer, Berlin, 1984.

12] , manuscript.

[13] S. Kerckhoff, The asymptotic geometry of Teichmüller space, Topology 19 (1980) 23-41.

[14] E. Reich, On the variational principle of Gerstenhaber and Rauch, Ann. Acad. Sci. Fenn. Ser. A I Math. 10 (1985) 469-475.

[15] H. L. Royden, Oral communication.

[16] __ Real analysis, 2nd ed., MacMillan, New York, 1968.

[17] J. H. Sampson, Some properties and applications of harmonic mappings, Ann. Sci. École Norm. Sup. 4 (1978) 211-228.

[18] R. Schoen \& S. T. Yau, On univalent harmonic maps between surfaces, Invent. Math. 44 (1978) 265-278.

[19] __ Existence of imcompressible minimal surfaces and the topology of three dimensional manifolds with nonnegative scalar curvature, Ann. of Math. (2) 110 (1979) 127-142.

[20] K. Strebel, Quadratic differentials, Springer, Berlin, 1984.

[21] B. Tabak, A geometric characterization of harmonic maps between surfaces, Math. Ann. 270 (1985) 147-157.

[22] W. Thurston, On the geometry and dynamics of diffeomorphisms of surfaces. I, preprint.

[23] A. J. Tromba, A new proof that Teichmüller's space is a cell, preprint.

[24] __ On a natural algebraic affine connection on the space of almost complex structures and the curvature of Teichmüller space with respect to its Weil-Petersson metric, preprint.

[25] M. Tsuji, Potential theory in modern function theory, Chelsea, New York, 1975.

[26] S. A. Wolpert, Non-completeness of the Weil-Petersson metric for Teichmuller space, $\mathrm{Pa}$ cific J. Math. 61 (1975) 573-57.

[27] _ Chern forms and the Riemann tensor for the moduli space of curves, Invent. Math. 85 (1986) 119-145.

[28] _ Geodesic length functions and the Nielsen problem, J. Differential Geometry 25 (1987) 275-296. 
